\title{
A two-layer approach to wave modelling
}

\author{
By Patrick Lynett $\dagger$ And Philip L.-F. LiU \\ School of Civil and Environmental Engineering, Cornell University, \\ Ithaca, NY 14853, USA (plynett@civil.tamu.edu) \\ Received 4 April 2003; revised 11 November 2003; accepted 24 February 2004; \\ published online 3 June 2004
}

\begin{abstract}
A set of model equations for water-wave propagation is derived by piecewise integration of the primitive equations of motion through two arbitrary layers. Within each layer, an independent velocity profile is derived. With two separate velocity profiles, matched at the interface of the two layers, the resulting set of equations has three free parameters, allowing for an optimization with known analytical properties of water waves. The optimized model equations show good linear wave characteristics up to $k h \approx 6$, while the second-order nonlinear behaviour is captured to $k h \approx 6$ as well. A numerical algorithm for solving the model equations is developed and tested against one- and two-horizontal-dimension cases. Agreement with laboratory data is excellent.
\end{abstract}

Keywords: nonlinear dispersive waves; landslide; Boussinesq

\section{Introduction}

The past decade saw the advent and widespread applications of Boussinesq-type equation models for studying water-wave propagation in one and two horizontal dimensions. This depth-integrated modelling approach employs a polynomial approximation of the vertical profile of the velocity field, thereby reducing the dimensions of a three-dimensional problem by one. The conventional Boussinesq equations (Peregrine 1967), which make use of a quadratic polynomial approximation for the vertical flow distribution, have two major constraints:

(i) the depth-averaged model describes the frequency dispersion of wave propagation in intermediate depths poorly;

(ii) the weakly nonlinear assumption limits the largest wave height that can accurately be modelled.

These constraints are consistent with the fundamental assumption of the Boussinesq equations, which states that leading-order dispersion and nonlinear effects are of the same order and are weak, i.e. $O\left(\mu_{0}^{2}\right)=O\left(\varepsilon_{0}\right) \ll 1$, where $\mu_{0}$ is equal to the wavenumber times depth $(k h)$ and $\varepsilon_{0}$ is the amplitude over depth $(a / h)$. The dispersive properties of the conventional Boussinesq equations have been improved by modifying the dispersive terms (Madsen \& Sørensen 1992) or using a reference velocity at a

$\dagger$ Present address: Department of Civil Engineering, Texas A\&M University, College Station, TX 77843-3136, USA.

Proc. R. Soc. Lond. A (2004) 460, 2637-2669

(C) 2004 The Royal Society 
specified depth (Nwogu 1993). These techniques yield a set of equations whose linear dispersion relation can be adjusted such that the resulting intermediate-depth dispersion characteristics are close to those of linear wave theory. Liu (1994) and Wei et al. (1995) extended Nwogu's approach to highly nonlinear waves, developing models that not only can be applied to intermediate water depth but also are capable of simulating wave propagation with strong nonlinear interaction, i.e. $\varepsilon_{0}=O(1)$. In general, these model equations contain accurate linear dispersion properties to $k h \approx 3$ (e.g. Nwogu 1993). In intermediate depths, nonlinear properties tend to exhibit larger relative errors than linear properties (Madsen \& Schäffer 1998), although additional enhancements can indeed create accurate nonlinear characteristics to near the linear accuracy limit, $k h \approx 3$ (Kennedy et al. 2001).

Further enhancing the deep-water accuracy of the depth-integrated approach are the so-called high-order Boussinesq-type equations. While the model equations described in the previous paragraph use a quadratic polynomial approximation for the vertical flow distribution, these high-order models use fourth- (and higher) order polynomial approximations. Gobbi et al. (2000), using a fourth-order polynomial, developed a model with excellent linear dispersive properties up to $k h \approx 6$. Nonlinear behaviour was faithfully captured to $k h \approx 3$. With the drastic improvement in accuracy over previous model equations comes a significant computational increase. The fourth-order polynomial employed results in fifth-order spatial derivatives in an extremely complex equation system, requiring an equally complex numerical scheme. Additionally, the complexity increases again for a two-horizontal dimension (2HD) problem, for which no high-order modelling attempts have yet been made. The reader is directed to Madsen \& Schäffer (1998), a thorough analysis of numerous different depth-integrated model equations, for additional information.

In this paper, a different approach to obtaining a high-order depth-integrated model is taken. Instead of employing a high-order polynomial approximation for the vertical distribution of the flow field, two quadratic polynomials are used, matched at an interface that divides the water column into two layers. This approach leads to a set of model equations without the high-order spatial derivatives associated with high-order polynomial approximations. The multi-layer concept has been attempted previously by Kanayama et al. (1998), although the derivation and final model equations are very different from those presented here. Internal wave and stratified flow modellers often employ a multi-layering concept, although the layers are of different density and thus represent a dissimilar physical problem to the one examined in this paper. Madsen et al. (2002) developed a model, based on the method of Agnon et al. (1999), accurate to extremely deep water $(k h \approx 40)$. Their derivation, fundamentally different from the one presented in this paper, involves optimal expansions of the Laplace equation, allowing for excellent deep-water linear and nonlinear dispersive properties of the resulting model. By using multiple expansions at various levels in the water column, the deep-water accuracy is achieved while only requiring the fifthorder spatial derivatives found in alternative high-order models with much smaller deep-water limitations. However, Madsen et al.'s model consists of more equations than the alternative models, and thus more unknowns. This is quite similar to the basic idea of the multi-layer derivation presented here: to trade fewer unknowns and higher spatial derivatives for more unknowns and lower spatial derivatives.

In $\S 2$ of this paper, the derivation of the two-layer, depth-integrated model is presented. Analysis of the model follows, including examination of linear disper- 


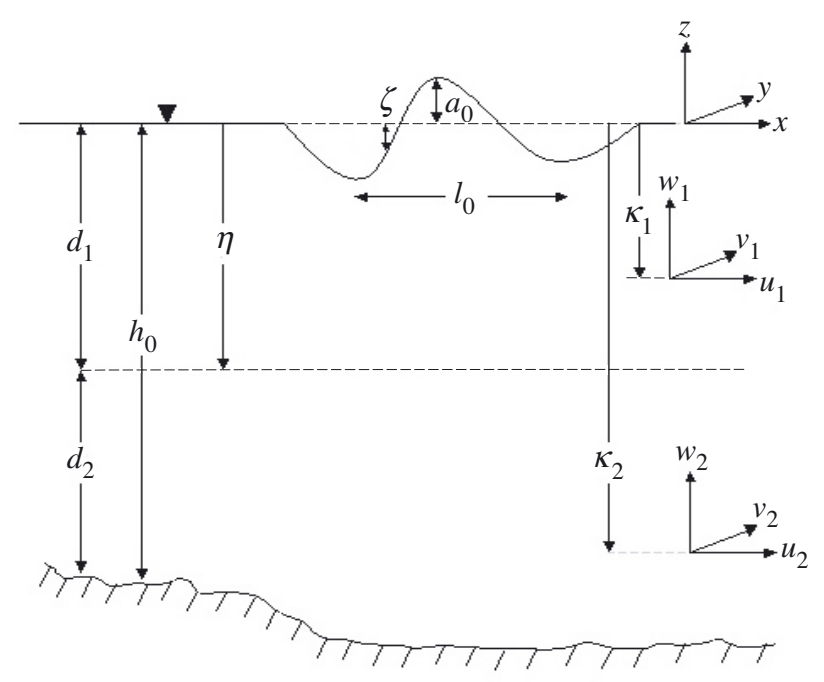

Figure 1. Problem set-up.

sion, shoaling, and nonlinear properties. These properties are optimized, based on agreements with linear and Stokes wave theories, and it is shown that the two-layer model is accurate into deep water. Finally, a numerical algorithm is developed for the general $2 \mathrm{HD}$ problem, and numerical solutions are compared with analytical and experimental data.

\section{Derivation of model equations}

\section{(a) Governing equations and boundary conditions}

The goal of this section is to derive a set of equations by integrating the primitive equations of motion in the vertical direction. It is noted that, although the derivation presented here employs just two layers, the procedure is directly extendable to any arbitrary number of layers (Lynett 2002). The flow region is divided by an interface, $z^{\prime}=\eta^{\prime}$, as shown in figure 1 . The resulting upper layer has a characteristic thickness $d_{1}$ (all variables contained entirely in this layer will be denoted with the subscript ' 1 '), while the lower layer has a thickness $d_{2}$ (variables in this layer denoted by the subscript ' 2 '). The determination of the location of the interface constitutes a part of the formulation of the model equations. Using the layer thicknesses $d_{1}$ and $d_{2}$ as the vertical length-scales in the upper and lower layer, respectively, the characteristic length of the wave $\ell_{0}$ as the horizontal length-scale, $h_{0}$ as the characteristic water depth, $\ell_{0} / \sqrt{g h_{0}}$ as the time-scale, and the characteristic wave amplitude $a_{0}$ as the scale of wave motion, we can define the dimensionless variables

$$
\left.\begin{array}{c}
(x, y)=\frac{\left(x^{\prime}, y^{\prime}\right)}{\ell_{0}}, \quad z_{n}=\frac{z^{\prime}}{d_{n}}, \quad t=\frac{\sqrt{g h_{0}} t^{\prime}}{\ell_{0}}, \\
h=\frac{h^{\prime}}{h_{0}}, \quad \zeta=\frac{\zeta^{\prime}}{a_{0}}, \quad \eta=\frac{\eta^{\prime}}{d_{1}}, \\
\left(U_{n}, V_{n}\right)=\frac{\left(U_{n}^{\prime}, V_{n}^{\prime}\right)}{\varepsilon_{0} \sqrt{g h_{0}}}, \quad W_{n}=\frac{W_{n}^{\prime}}{\varepsilon_{0} \mu_{0} \sqrt{g h_{0}}}, \quad p_{n}=\frac{p^{\prime}}{\rho g a_{0}},
\end{array}\right\}
$$


in which the subscript $n=1,2$ indicates the layer index, $\left(U_{n}, V_{n}\right)$ represent the horizontal velocity components in the different layers, $W_{n}$ the vertical velocity component, and $p_{n}$ the pressure. Note that the vertical coordinate, $z_{n}$, is scaled differently in each layer. Two dimensionless parameters have been introduced in (2.1), i.e.

$$
\varepsilon_{0}=\frac{a_{0}}{h_{0}}, \quad \mu_{0}=\frac{h_{0}}{\ell_{0}} .
$$

Assuming that the viscous effects are insignificant, the wave motion can be described by the continuity equation and the Euler's equations, i.e.

$$
\begin{gathered}
\frac{d_{n}}{h_{0}} \nabla \cdot \boldsymbol{U}_{n}+\frac{\partial W_{n}}{\partial z_{n}}=0 \\
\frac{\partial \boldsymbol{U}_{n}}{\partial t}+\varepsilon_{0} \boldsymbol{U}_{n} \cdot \nabla \boldsymbol{U}_{n}+\varepsilon_{n} W_{n} \frac{\partial \boldsymbol{U}_{n}}{\partial z_{n}}=-\nabla p_{n} \\
\mu_{n}^{2}\left(\frac{\partial W_{n}}{\partial t}+\varepsilon_{0} \boldsymbol{U}_{n} \cdot \nabla W_{n}\right)+\varepsilon_{0} \mu_{0}^{2} W_{n} \frac{\partial W_{n}}{\partial z_{n}}=-\left(\frac{\partial p_{n}}{\partial z_{n}}+\frac{1}{\varepsilon_{n}}\right),
\end{gathered}
$$

where

$$
\mu_{n}^{2}=\frac{d_{n} h_{0}}{l_{0}^{2}}, \quad \varepsilon_{n}=\frac{a_{0}}{d_{n}},
$$

$\boldsymbol{U}_{n}=\left(U_{n}, V_{n}\right)$ denotes the horizontal velocity vector, and $\nabla=(\partial / \partial x, \partial / \partial y)$ the horizontal gradient vector.

On the free surface, $z_{1}=\varepsilon_{1} \zeta(x, y, t)$, the usual kinematic and dynamic boundary condition applies:

$$
\begin{array}{cc}
W_{1}=\frac{\partial \zeta}{\partial t}+\varepsilon_{0} \boldsymbol{U}_{1} \cdot \nabla \zeta & \text { on } z_{1}=\varepsilon_{1} \zeta \\
p_{1}=0 & \text { on } z_{1}=\varepsilon_{1} \zeta .
\end{array}
$$

Along the seafloor,

$$
z_{2}=-\frac{h_{0}}{d_{2}} h
$$

the kinematic boundary condition requires

$$
W_{2}+\boldsymbol{U}_{2} \cdot \nabla h+\frac{1}{\varepsilon_{0}} \frac{\partial h}{\partial t}=0 \quad \text { on } z_{2}=-\frac{h_{0}}{d_{2}} h .
$$

Note that due to the assumption that $h=h(x, y, t)$, the time derivative of $h$ appears in the bottom boundary condition. This assumption will allow for the study of waves generated by seafloor movements, such as underwater landslides. At the interface between the layers, continuity of pressure and velocity is required:

$$
\begin{aligned}
& p_{1}=p_{2} \quad \text { on } z_{1}=\eta, \quad z_{2}=\frac{d_{1}}{d_{2}} \eta, \\
& \boldsymbol{U}_{1}=\boldsymbol{U}_{2} \quad \text { on } z_{1}=\eta, \quad z_{2}=\frac{d_{1}}{d_{2}} \eta, \\
& W_{1}=W_{2} \quad \text { on } z_{1}=\eta, \quad z_{2}=\frac{d_{1}}{d_{2}} \eta \text {. }
\end{aligned}
$$


For later use, we note here that the exact depth-integrated continuity equation can be obtained by integrating (2.3) across each of the layers. After applying the boundary conditions $(2.6),(2.8)$ and $(2.11)$, the resulting equation reads

$$
\nabla \cdot\left[\frac{d_{1}}{h_{0}} \int_{\eta}^{\varepsilon_{1} \zeta} \boldsymbol{U}_{1} \mathrm{~d} z_{1}+\frac{d_{2}}{h_{0}} \int_{-h_{0} h / d_{2}}^{d_{1} \eta / d_{2}} \boldsymbol{U}_{2} \mathrm{~d} z_{2}\right]+\frac{1}{\varepsilon_{0}} \frac{\partial h}{\partial t}+\frac{\partial \zeta}{\partial t}=0 .
$$

(b) Approximate two-dimensional governing equations

A perturbation analysis will be performed using the assumption

$$
O\left(\mu_{1}^{2}\right)=O\left(\mu_{2}^{2}\right) \ll 1 .
$$

Using $\mu_{n}^{2}$ as the small parameter, we can expand the dimensionless physical variables as power series of $\mu_{n}^{2}$ :

$$
f=\sum_{M=0}^{\infty} \mu_{n}^{2 M} f^{[M]} ;\left(f=\boldsymbol{U}_{n}, W_{n}, \zeta, p_{n}\right) .
$$

Furthermore, we will assume the flow is irrotational. Zero horizontal vorticity yields the conditions

$$
\begin{aligned}
\frac{\partial}{\partial z_{n}} \boldsymbol{U}_{n}^{[0]} & =0, \\
\frac{\partial}{\partial z_{n}} \boldsymbol{U}_{n}^{[1]} & =\nabla W_{n}^{[0]} .
\end{aligned}
$$

Consequently, from (2.15), the leading-order horizontal velocity components are independent of the vertical coordinate, i.e.

$$
\boldsymbol{U}_{n}^{[0]}=\boldsymbol{U}_{n}^{[0]}(x, y, t)
$$

Substituting (2.14) into the continuity equation (2.3) and the boundary conditions (2.6) and (2.8), we collect the leading-order terms as

$$
\begin{gathered}
\frac{d_{n}}{h_{0}} \nabla \cdot \boldsymbol{U}_{n}^{[0]}+\frac{\partial W_{n}^{[0]}}{\partial z_{n}}=0, \quad-\frac{h_{0}}{d_{2}} h<z_{2}<\frac{d_{1}}{d_{2}} \eta, \quad \eta<z_{1}<\varepsilon_{1} \zeta, \\
W_{1}^{[0]}=\frac{\partial \zeta}{\partial t}+\varepsilon_{0} \boldsymbol{U}_{1}^{[0]} \cdot \nabla \zeta \quad \text { on } z_{1}=\varepsilon_{1} \zeta, \\
W_{2}^{[0]}+\boldsymbol{U}_{2}^{[0]} \cdot \nabla h+\frac{1}{\varepsilon_{0}} \frac{\partial h}{\partial t}=0 \quad \text { on } z_{2}=-\frac{h_{0}}{d_{2}} h .
\end{gathered}
$$

Integrating (2.18) with respect to $z_{n}$ and using (2.20) to determine the integration constant, we obtain the vertical profile of the vertical velocity components in the layers,

$$
\begin{aligned}
& W_{2}^{[0]}=-z_{2} S_{2}^{[0]}-T_{2}^{[0]}, \\
& W_{1}^{[0]}=-z_{1} S_{1}^{[0]}-T_{1}^{[0]},
\end{aligned}
$$


where

$$
\left.\begin{array}{ll}
S_{2}^{[0]}=\frac{d_{2}}{h_{0}} \nabla \cdot \boldsymbol{U}_{2}^{[0]}, & T_{2}^{[0]}=\nabla \cdot\left(h \boldsymbol{U}_{2}^{[0]}\right)+\frac{1}{\varepsilon_{0}} \frac{\partial h}{\partial t}, \\
S_{1}^{[0]}=\frac{d_{1}}{h_{0}} \nabla \cdot \boldsymbol{U}_{1}^{[0]}, & T_{1}^{[0]}=\eta\left(\frac{d_{1}}{d_{2}} S_{2}^{[0]}-S_{1}^{[0]}\right)+T_{2}^{[0]} \cdot
\end{array}\right\}
$$

Similarly, by integrating (2.16) in $z_{n}$ with information from (2.21) and (2.22), we can find the corresponding vertical profiles of the horizontal velocity components,

$$
\boldsymbol{U}_{n}^{[1]}=-\frac{z_{n}^{2}}{2} \nabla S_{n}^{[0]}-z_{n} \nabla T_{n}^{[0]}+\boldsymbol{C}_{n}(x, y, t),
$$

in which $\boldsymbol{C}_{n}$ are unknown functions. Up to $O\left(\mu_{n}^{2}\right)$, the horizontal velocity components can be expressed as

$$
\boldsymbol{U}_{n}=\boldsymbol{U}_{n}^{[0]}-\mu_{n}^{2}\left\{\frac{1}{2} z_{n}^{2} \nabla S_{n}^{[0]}+z_{n} \nabla T_{n}^{[0]}+\boldsymbol{C}_{n}\right\}+O\left(\mu_{n}^{4}\right) .
$$

Now, we can define the horizontal velocity vectors, $\boldsymbol{u}_{n}\left(x, y, \kappa_{n}(x, y, t), t\right)$ evaluated at $z_{n}=\kappa_{n}(x, y, t)$ as

$$
\boldsymbol{u}_{n}=\boldsymbol{U}_{n}^{[0]}-\mu_{n}^{2}\left\{\frac{1}{2} \kappa_{n}^{2} \nabla S_{n}^{[0]}+\kappa_{n} \nabla T_{n}^{[0]}+\boldsymbol{C}_{n}\right\}+O\left(\mu_{n}^{4}\right) .
$$

Subtracting (2.26) from (2.25) we can express $\boldsymbol{U}_{n}$ in terms of $\boldsymbol{u}_{n}$ as

$$
\begin{aligned}
& \boldsymbol{U}_{2}=\boldsymbol{u}_{2}-\mu_{2}^{2}\left\{\frac{1}{2}\left(z_{2}^{2}-\kappa_{2}^{2}\right) \nabla S_{2}+\left(z_{2}-\kappa_{2}\right) \nabla T_{2}\right\}+O\left(\mu_{2}^{4}\right), \\
& \boldsymbol{U}_{1}=\boldsymbol{u}_{1}-\mu_{1}^{2}\left\{\frac{1}{2}\left(z_{1}^{2}-\kappa_{1}^{2}\right) \nabla S_{1}+\left(z_{1}-\kappa_{1}\right) \nabla T_{1}\right\}+O\left(\mu_{1}^{4}, \mu_{1}^{2} \mu_{2}^{2}\right),
\end{aligned}
$$

where

$$
\left.\begin{array}{ll}
S_{2}=\frac{d_{2}}{h_{0}} \nabla \cdot \boldsymbol{u}_{2}, & T_{2}=\nabla \cdot\left(h \boldsymbol{u}_{2}\right)+\frac{1}{\varepsilon_{0}} \frac{\partial h}{\partial t}, \\
S_{1}=\frac{d_{1}}{h_{0}} \nabla \cdot \boldsymbol{u}_{1}, & T_{1}=\eta\left(\frac{d_{1}}{d_{2}} S_{2}-S_{1}\right)+T_{2},
\end{array}\right\}
$$

Note that $\boldsymbol{u}_{n}=\boldsymbol{U}_{n}^{[0]}+O\left(\mu_{n}^{2}\right)$ has been used in (2.27) and (2.28).

The pressure field is determined by first approximating the vertical momentum equation (2.5) as

$$
\begin{gathered}
\frac{\partial p_{n}}{\partial z_{n}}=-\frac{1}{\varepsilon_{n}}-\mu_{n}^{2}\left(\frac{\partial W_{n}^{[0]}}{\partial t}+\varepsilon_{0} \boldsymbol{U}_{n}^{[0]} \cdot \nabla W_{n}^{[0]}\right)-\mu_{0}^{2}\left(\varepsilon_{0} W_{n}^{[0]} \frac{\partial W_{n}^{[0]}}{\partial z_{n}}\right)+O\left(\mu_{0}^{2} \mu_{n}^{2}, \mu_{n}^{4}\right), \\
-\frac{h_{0}}{d_{2}} h<z_{2}<\frac{d_{1}}{d_{2}} \eta \quad \text { for } n=2, \quad \eta<z_{1}<\varepsilon_{1} \zeta \quad \text { for } n=1 .
\end{gathered}
$$

We can integrate the equation above with respect to $z_{1}$ to find the pressure field in the upper layer as

$$
\begin{aligned}
p_{1}=\zeta & -\frac{z_{1}}{\varepsilon_{1}}+\mu_{1}^{2}\left\{\frac{1}{2}\left(z_{1}^{2}-\varepsilon_{1}^{2} \zeta^{2}\right) \frac{\partial S_{1}}{\partial t}+\left(z_{1}-\varepsilon_{1} \zeta\right) \frac{\partial T_{1}}{\partial t}\right. \\
& \left.+\frac{1}{2} \varepsilon_{0}\left(z_{1}^{2}-\varepsilon_{1}^{2} \zeta^{2}\right) \boldsymbol{u}_{1} \cdot \nabla S_{1}+\varepsilon_{0}\left(z_{1}-\varepsilon_{1} \zeta\right) \boldsymbol{u}_{1} \cdot \nabla T_{1}\right\} \\
& +\varepsilon_{0} \mu_{0}^{2}\left\{\frac{1}{2}\left(\varepsilon_{1}^{2} \zeta^{2}-z_{1}^{2}\right) S_{1}^{2}+\left(\varepsilon_{1} \zeta-z_{1}\right) S_{1} T_{1}\right\}+O\left(\mu_{0}^{2} \mu_{n}^{2}, \mu_{n}^{4}\right), \quad \eta<z_{1}<\varepsilon_{1} \zeta .
\end{aligned}
$$


To derive the governing equations for $\boldsymbol{u}_{1}$, we substitute (2.28) and (2.31) into (2.4), enforce zero vertical vorticity (see Hsiao \& Liu 2002), and obtain

$$
\begin{gathered}
\frac{\partial \boldsymbol{u}_{1}}{\partial t}+\frac{1}{2} \varepsilon_{0} \nabla\left(\boldsymbol{u}_{1} \cdot \boldsymbol{u}_{1}\right)+\nabla \zeta+\mu_{1}^{2} \frac{\partial}{\partial t}\left\{\frac{1}{2} \kappa_{1}^{2} \nabla S_{1}+\kappa_{1} \nabla T_{1}\right\} \\
+\varepsilon_{0} \mu_{1}^{2} \nabla\left(\kappa_{1} \boldsymbol{u}_{1} \cdot \nabla T_{1}+\frac{1}{2} \kappa_{1}^{2} \boldsymbol{u}_{1} \cdot \nabla S_{1}\right)+\varepsilon_{0} \mu_{0}^{2}\left[T_{1} \nabla T_{1}-\nabla\left(\zeta \frac{\partial T_{1}}{\partial t}\right)\right] \\
+\varepsilon_{0}^{2} \mu_{0}^{2} \nabla\left(\zeta S_{1} T_{1}-\frac{h_{0}}{d_{1}} \frac{\zeta^{2}}{2} \frac{\partial S_{1}}{\partial t}-\zeta \boldsymbol{u}_{1} \cdot \nabla T_{1}\right) \\
+\varepsilon_{0}^{2} \varepsilon_{1} \mu_{0}^{2} \nabla\left[\frac{\zeta^{2}}{2}\left(S_{1}^{2}-\frac{h_{0}}{d_{1}} \boldsymbol{u}_{1} \cdot \nabla S_{1}\right)\right]=O\left(\mu_{0}^{2} \mu_{1}^{2}\right) .
\end{gathered}
$$

Note that $\varepsilon_{0} \mu_{0}^{2}=\varepsilon_{1} \mu_{1}^{2}$, and all coefficients in the above equation are written in terms of $\mu_{0}$ and $\varepsilon_{0}$ whenever possible.

With boundary condition (2.10) and the known velocity profiles (2.27) and (2.28), $\boldsymbol{u}_{2}$ can be expressed as a function of $\boldsymbol{u}_{1}$ :

$$
\begin{aligned}
\boldsymbol{u}_{2}+\mu_{2}^{2}\left\{\frac{1}{2}\left(\kappa_{2}^{2}-\frac{d_{1}^{2}}{d_{2}^{2}} \eta^{2}\right) \nabla S_{2}+\left(\kappa_{2}-\frac{d_{1}}{d_{2}} \eta\right) \nabla T_{2}\right\} \\
=\boldsymbol{u}_{1}+\mu_{1}^{2}\left\{\frac{1}{2}\left(\kappa_{1}^{2}-\eta^{2}\right) \nabla S_{1}+\left(\kappa_{1}-\eta\right) \nabla T_{1}\right\}+O\left(\mu_{1}^{4}, \mu_{1}^{2} \mu_{2}^{2}, \mu_{2}^{4}\right) .
\end{aligned}
$$

Thus, the lower-layer velocity can be directly calculated with knowledge of the upperlayer velocity.

The exact continuity equation (2.12) can be rewritten approximately in terms of $\zeta$ and $\boldsymbol{u}_{n}$. Substituting (2.27) into (2.12), we obtain

$$
\begin{gathered}
\frac{h_{0}}{d_{1} \varepsilon_{0}} \frac{\partial h}{\partial t}+\frac{h_{0}}{d_{1}} \frac{\partial \zeta}{\partial t}+\nabla \cdot\left[\left(\varepsilon_{1} \zeta-\eta\right) \boldsymbol{u}_{1}+\left(\eta+\frac{h_{0}}{d_{1}} h\right) \boldsymbol{u}_{2}\right] \\
-\mu_{2}^{2} \frac{d_{2}}{d_{1}} \nabla \cdot\left\{\left[\frac{1}{6}\left(\eta^{3} \frac{d_{1}^{3}}{d_{2}^{3}}+h^{3} \frac{h_{0}^{3}}{d_{2}^{3}}\right)-\frac{1}{2}\left(\eta \frac{d_{1}}{d_{2}}+h \frac{h_{0}}{d_{2}}\right) \kappa_{2}^{2}\right] \nabla S_{2}\right. \\
\left.+\left[\frac{1}{2}\left(\eta^{2} \frac{d_{1}^{2}}{d_{2}^{2}}-h^{2} \frac{h_{0}^{2}}{d_{2}^{2}}\right)-\left(\eta \frac{d_{1}}{d_{2}}+h \frac{h_{0}}{d_{2}}\right) \kappa_{2}\right] \nabla T_{2}\right\} \\
-\mu_{1}^{2} \nabla \cdot\left\{\left[\frac{1}{6}\left(\varepsilon_{1}^{3} \zeta^{3}-\eta^{3}\right)-\frac{1}{2}\left(\varepsilon_{1} \zeta-\eta\right) \kappa_{1}^{2}\right] \nabla S_{1}+\left[\frac{1}{2}\left(\varepsilon_{1}^{2} \zeta^{2}-\eta^{2}\right)-\left(\varepsilon_{1} \zeta-\eta\right) \kappa_{1}\right] \nabla T_{1}\right\} \\
=O\left(\mu_{1}^{4}, \mu_{1}^{2} \mu_{2}^{2}, \mu_{2}^{4}\right) .
\end{gathered}
$$

Equations (2.32)-(2.34) are the coupled governing equations, written in terms of $\boldsymbol{u}_{n}$ and $\zeta$, for highly nonlinear, dispersive waves.

We remark here that the matching conditions along the interface, (2.10) and (2.11), do not require the continuity of the vertical velocity gradients. Consequently, this leads to a discontinuity of the nonlinear vertical transport terms in the horizontal and vertical Euler equations. Specifically, the discontinuity arises in the $\varepsilon_{n} W_{n}\left(\partial \boldsymbol{U}_{n} / \partial z_{n}\right)$ term in (2.4) and the $\varepsilon_{0} \mu_{0}^{2} W_{n}\left(\partial W_{n} / \partial z_{n}\right)$ term in (2.5). However, with calculation of these nonlinear terms using the derived vertical velocity profiles, (2.21), (2.22), and horizontal velocity profiles, $(2.27),(2.28)$, it can be shown that the discontinuity is 
of the truncation error order in the final model, (2.32)-(2.34), i.e.

$$
\begin{gathered}
\frac{\partial \boldsymbol{U}_{1}\left(z_{1}=\eta\right)}{\partial z_{1}}=\frac{\partial \boldsymbol{U}_{2}\left(z_{2}=d_{1} \eta / d_{2}\right)}{\partial z_{2}}+O\left(\mu_{1}^{4}, \mu_{1}^{2} \mu_{2}^{2}, \mu_{2}^{4}\right), \\
\mu_{0}^{2} \frac{\partial W_{1}\left(z_{1}=\eta\right)}{\partial z_{1}}=\mu_{0}^{2} \frac{\partial W_{2}\left(z_{2}=d_{1} \eta / d_{2}\right)}{\partial z_{2}}+O\left(\mu_{0}^{2} \mu_{1}^{2}, \mu_{0}^{2} \mu_{2}^{2}\right) .
\end{gathered}
$$

Thus, the discontinuity of the nonlinear, vertical transport terms will not affect the overall accuracy of the model equations.

\section{Analysis of model equations}

In this section, the properties of the two-layer model equations will be scrutinized and optimized. First, it is shown that the two-layer model equations reduce to the well-studied, 'extended' Boussinesq model derived by Nwogu (1993) after some $O\left(\mu_{n}^{2}\right)$ manipulation. With the use of $O\left(\mu_{n}^{2}\right)$ substitutions, namely

$$
\boldsymbol{u}_{2}=\boldsymbol{u}_{1}+O\left(\mu_{n}^{2}\right)
$$

we can eliminate one of the unknowns from our equation system. Rewriting (2.34) in terms of $\boldsymbol{u}_{1}$ only, assigning $d_{1}=h_{0}, \kappa_{2}=-\left(h_{0} / d_{2}\right) h, \eta=-h$, and examining the weakly nonlinear form of the equations, gives

$$
\begin{aligned}
& O\left(\varepsilon_{0} \mu_{0}^{2}, \mu_{0}^{4}\right) \\
& =\frac{1}{\varepsilon_{0}} \frac{\partial h}{\partial t}+\frac{\partial \zeta}{\partial t}+\nabla \cdot\left[\left(\varepsilon_{0} \zeta+h\right) \boldsymbol{u}_{1}\right] \\
& \quad-\mu_{0}^{2} \nabla \cdot\left\{\left[\frac{1}{6} h^{3}-\frac{1}{2} h \kappa_{1}^{2}\right] \nabla\left(\nabla \cdot \boldsymbol{u}_{1}\right)-\left[\frac{1}{2} h^{2}+h \kappa_{1}\right] \nabla\left[\nabla \cdot\left(h \boldsymbol{u}_{1}\right)+\frac{1}{\varepsilon_{0}} \frac{\partial h}{\partial t}\right]\right\} .
\end{aligned}
$$

The momentum equation (2.32) becomes

$$
\begin{aligned}
O\left(\varepsilon_{0} \mu_{0}^{2}, \mu_{0}^{4}\right)=\frac{\partial \boldsymbol{u}_{1}}{\partial t}+\varepsilon_{0} \boldsymbol{u}_{1} & \cdot \nabla \boldsymbol{u}_{1}+\nabla \zeta \\
+ & \mu_{0}^{2} \frac{\partial}{\partial t}\left\{\frac{\kappa_{1}^{2}}{2} \nabla\left(\nabla \cdot \boldsymbol{u}_{1}\right)+\kappa_{1} \nabla\left[\nabla \cdot\left(h \boldsymbol{u}_{1}\right)+\frac{1}{\varepsilon_{0}} \frac{\partial h}{\partial t}\right]\right\} .
\end{aligned}
$$

This system for $\zeta$ and $\boldsymbol{u}_{1}$ is identical to the model derived by Nwogu, except for the addition of the $\partial h / \partial t$ terms. Additionally, the nonlinear dispersive terms, which have been truncated for the sake of brevity in (3.2) and (3.3), are identical to those derived by Liu (1994) and later implemented numerically by Lynett \& Liu (2002). For the rest of this paper, the 'extended' Boussinesq model including all the nonlinear dispersive terms up to $O\left(\mu_{0}^{2}\right)$, as given by Liu (1994), will be referred to as the one-layer model.

For the rest of this section, the focus will be on analysis of the three-unknown $\left(\zeta, \boldsymbol{u}_{1}, \boldsymbol{u}_{2}\right)$, two-layer system. Additionally, for the rest of this paper, all variables discussed are in dimensional form, with primes no longer applied. The momentum 
equation, (2.32), can be expressed in a more compact, dimensional form:

$$
\begin{gathered}
\frac{\partial \boldsymbol{u}_{1}}{\partial t}+\frac{1}{2} \nabla\left(\boldsymbol{u}_{1} \cdot \boldsymbol{u}_{1}\right)+g \nabla \zeta+\frac{\partial}{\partial t}\left\{\frac{1}{2} \kappa_{1}^{2} \nabla S_{1}+\kappa_{1} \nabla T_{1}-\nabla\left(\frac{1}{2} \zeta^{2} S_{1}\right)-\nabla\left(\zeta T_{1}\right)\right\} \\
+\nabla\left\{\frac{\partial \zeta}{\partial t}\left(T_{1}+\zeta S_{1}\right)+\left(\kappa_{1}-\zeta\right)\left(\boldsymbol{u}_{1} \cdot \nabla\right) T_{1}\right. \\
\left.+\frac{1}{2}\left(\kappa_{1}^{2}-\zeta^{2}\right)\left(\boldsymbol{u}_{1} \cdot \nabla\right) S_{1}+\frac{1}{2}\left[\left(T_{1}+\zeta S_{1}\right)^{2}\right]\right\}=0 .
\end{gathered}
$$

This is the momentum equation that will be analysed and numerically solved in this paper. Before solving the system numerically, the linear and nonlinear dispersion properties are examined for the case of unidirectional propagation in water of constant depth. Let us define the arbitrary evaluation levels and the boundary between the two layers as

$$
\kappa_{1}=\alpha_{1} h+\beta_{1} \zeta, \quad \eta=\alpha_{2} h+\beta_{2} \zeta, \quad \kappa_{2}=\alpha_{3} h+\beta_{3} \zeta,
$$

where the coefficients $\alpha$ and $\beta$ are arbitrary and to be defined. From (3.4), the onehorizontal-dimension, constant-water-depth, two-layer equations can be obtained, which are given in Appendix A, and will be used to determine the linear and nonlinear properties of the two-layer model. The solution of the one-dimensional equations takes the form

$$
\left.\begin{array}{r}
\zeta=\epsilon \zeta^{(0)} \mathrm{e}^{\mathrm{i} \theta}+\epsilon^{2} \zeta^{(1)} \mathrm{e}^{2 \mathrm{i} \theta}+\cdots, \\
\boldsymbol{u}_{1}=\epsilon \boldsymbol{u}_{1}^{(0)} \mathrm{e}^{\mathrm{i} \theta}+\epsilon^{2} \boldsymbol{u}_{1}^{(1)} \mathrm{e}^{2 \mathrm{i} \theta}+\cdots, \\
\boldsymbol{u}_{2}=\epsilon \boldsymbol{u}_{2}^{(0)} \mathrm{e}^{\mathrm{i} \theta}+\epsilon^{2} \boldsymbol{u}_{2}^{(1)} \mathrm{e}^{2 \mathrm{i} \theta}+\cdots
\end{array}\right\}
$$

where $\theta=k x-w t, k$ is the wavenumber, $w$ is the wave frequency and $\epsilon$ is simply an ordering parameter. In following sections, the form of above solution will be used to determine the linear dispersion, linear shoaling and leading-order nonlinear properties of the two-layer model equations. Only the formulae will be presented in the body of the paper, with derivation details presented in Appendix A.

\section{(a) Linear dispersion relation}

After substituting (3.6) into the governing equations (2.32)-(2.34), the first order (in $\epsilon$ ) system yields the linear dispersion relation

$$
c^{2}=\frac{w^{2}}{k^{2}}=\frac{g h\left[1+N_{1}(k h)^{2}+N_{2}(k h)^{4}\right]}{1+D_{1}(k h)^{2}+D_{2}(k h)^{4}},
$$

where $c$ is the wave celerity and the coefficients $N_{1}, N_{2}, D_{1}$ and $D_{2}$ are given in Appendix A $a$ and are solely functions of $\alpha_{1}, \alpha_{2}$ and $\alpha_{3}$. The above dispersion relation will be compared with the $[4,4]$ Padé approximation

$$
c^{2}=\frac{w^{2}}{k^{2}}=\frac{g h\left[1+\frac{1}{9}(k h)^{2}+\frac{1}{945}(k h)^{4}\right]}{1+\frac{4}{9}(k h)^{2}+\frac{1}{63}(k h)^{4}}
$$

of the exact linear dispersion relation,

$$
c_{\mathrm{e}}^{2}=\frac{w^{2}}{k^{2}}=\frac{g}{k} \tanh (k h) .
$$

Proc. R. Soc. Lond. A (2004) 
The Padé approximation used here is approximation of $\tanh (k h) / k h$, where the numbers in the Padé brackets represent the highest polynomial order of $k h$ in the numerator and denominator. The group velocity of the two-layer model equations, $c_{\mathrm{g}}$, can be determined straightforwardly by taking the derivative of (3.7) with respect to $k$.

\section{(b) Vertical velocity profiles}

Let us define the function $f_{1}(z)$ as the horizontal velocity profile, with constant water depth, normalized by its value at $z=0$. This function is derived from the substitution of (3.6) into (2.27) and (2.28), and is composed of two quadratic polynomial elements, given by

$$
f_{1}(z)= \begin{cases}\frac{1+(k h)^{2}\left[\frac{1}{2}\left(z^{2} / h^{2}-\alpha_{1}^{2}\right)+\alpha_{2}\left(\alpha_{1}-z / h\right)\right.}{1-(k h)^{2}\left[\frac{1}{2} \alpha_{1}^{2}-\alpha_{2} \alpha_{1}+u_{2}^{(0)} / u_{1}^{(0)}\left(\alpha_{2}+1\right) \alpha_{1}\right]} & \text { for } z \geqslant \eta=\alpha_{2} h, \\ f_{1}(\eta) \frac{1+(k h)^{2}\left[\frac{1}{2}\left(z^{2} / h^{2}-\alpha_{3}^{2}\right)+\left(z / h-\alpha_{3}\right)\right]}{1+(k h)^{2}\left[\frac{1}{2}\left(\alpha_{2}^{2}-\alpha_{3}^{2}\right)+\left(\alpha_{2}-\alpha_{3}\right)\right]} & \text { for } z<\eta=\alpha_{2} h .\end{cases}
$$

From the linear-equation system we can also find that

$$
\begin{aligned}
u_{1}^{(0)} & =\frac{g \zeta^{(0)}\left[k h-\delta_{8}(k h)^{3}\right]}{h w\left[1+D_{1}(k h)^{2}+D_{2}(k h)^{4}\right]}, \\
u_{2}^{(0)} & =\frac{g \zeta^{(0)}\left[k h+\delta_{7}(k h)^{3}\right]}{h w\left[1+D_{1}(k h)^{2}+D_{2}(k h)^{4}\right]},
\end{aligned}
$$

where $\delta_{7}$ and $\delta_{8}$ are given in Appendix A, and thus the ratio $u_{2}^{(0)} / u_{1}^{(0)}$ present in (3.10) can be evaluated. Similarly, the vertical profile of the vertical velocity component, normalized by its value at the still water level, is given by

$$
f_{2}(z)= \begin{cases}\frac{z / h-\alpha_{2}+u_{2}^{(0)} / u_{1}^{(0)}\left(\alpha_{2}+1\right)}{-\alpha_{2}+u_{2}^{(0)} / u_{1}^{(0)}\left(\alpha_{2}+1\right)} & \text { for } z \geqslant \eta=\alpha_{2} h, \\ f_{2}(\eta) \frac{z / h+1}{\alpha_{2}+1} & \text { for } z<\eta=\alpha_{2} h,\end{cases}
$$

which is a piecewise linear function.

\section{(c) Linear shoaling properties}

Based on linear theory, the exact shoaling gradient is given as

$$
\frac{a_{x}^{\mathrm{e}}}{a}=A_{x}^{\mathrm{e}} \frac{h_{x}}{h}=-k h \tanh (k h) \frac{[1-k h \tanh (k h)]\left[1-\tanh ^{2}(k h)\right]}{\left\{\tanh (k h)+k h\left[1-\tanh ^{2}(k h)\right]\right\}^{2}} \frac{h_{x}}{h} .
$$

The linear shoaling properties of the present two-layer model are expressed as

$$
\frac{a_{x}}{a}=A_{x} \frac{h_{x}}{h} .
$$

The determination of $A_{x}$ follows the procedure given by Schäffer \& Madsen (1995), and is described in Appendix $\mathrm{A} b$. 
(d) Second-order, nonlinear interactions: steady waves

Now, the nonlinear corrections to the linear problem are sought. Collecting $O\left(\epsilon^{2}\right)$ terms from the governing equations yields the second-order free-surface correction,

$$
\zeta^{(1)}=k \zeta^{(0)^{2}} L_{5},
$$

where the solution procedure for $L_{5}$ is described in Appendix A $c$. This approximate expression can be compared with the second-order Stokes wave solution:

$$
\zeta_{\text {Stokes }}^{(1)}=\frac{1}{4} k \zeta^{(0)^{2}}\left[3 \operatorname{coth}^{3}(k h)-\operatorname{coth}(k h)\right] .
$$

(e) Second-order, nonlinear interactions: bichromatic interactions

Examining a two-wave group, the free surface can be written as

$$
\begin{aligned}
\zeta=\epsilon \zeta_{1}^{(0)} \mathrm{e}^{\mathrm{i}\left(k_{1} x-w_{1} t\right)} & +\epsilon \zeta_{2}^{(0)} \mathrm{e}^{\mathrm{i}\left(k_{2} x-w_{2} t\right)}+\epsilon^{2} \zeta_{1}^{(1)} e^{2 i\left(k_{1} x-w_{1} t\right)} \\
& +\epsilon^{2} \zeta_{2}^{(1)} \mathrm{e}^{2 \mathrm{i}\left(k_{2} x-w_{2} t\right)}+\epsilon^{2} \zeta^{+} \mathrm{e}^{\mathrm{i}\left(k_{+} x-w_{+} t\right)}+\epsilon^{2} \zeta^{-} \mathrm{e}^{\mathrm{i}\left(k_{-} x-w_{-} t\right)},
\end{aligned}
$$

where $\zeta^{+}$and $\zeta^{-}$are the sum and difference components of the two first-order wave frequencies, $w_{\mp}=w_{1} \mp w_{2}$, and wavenumbers, $k_{\mp}=k_{1} \mp k_{2}$. Similar expressions can be given for $\boldsymbol{u}_{n}$. The bichromatic solution form is substituted into our model equations, allowing the determination of $\zeta^{\mp}$. The derivation method for $\zeta^{\mp}$ is given in Appendix A $c$. The sum and difference free-surface components can be compared with those from Stokes theory, $\zeta_{\text {Stokes }}^{\mp}$, which can be found in Schäffer (1996).

\section{(f) Choice of arbitrary levels: linear optimization}

Through examination of linear and nonlinear properties, the most accurate set of arbitrary levels will be chosen in this section. First, the linear properties of the twolayer model will be optimized, independent of nonlinearity. In the linear sense, the three levels are given as $\kappa_{1}=\alpha_{1} h, \eta=\alpha_{2} h$ and $\kappa_{2}=\alpha_{3} h$, where $\kappa_{1}$ and $\kappa_{2}$ are the levels at which horizontal velocities are evaluated in the upper and lower layers, and $\eta$ is the location of the interface between the layers. Of course, possible values are bounded by $0 \geqslant \alpha_{1} \geqslant \alpha_{2} \geqslant \alpha_{3} \geqslant-1$. Defining a model accuracy, or model error, can be difficult and often can depend on the specific physical problem being examined. For this analysis, a representation of the overall error, including errors in wave speed, group velocity and shoaling, is sought. The error will be given by the minimization parameter $\Delta_{\text {lin }}$ :

$$
\begin{aligned}
\Delta_{\text {lin }}=\frac{1}{3}\left(\frac{\sum_{k h=0.1}^{\Omega}\left(\left|c^{\mathrm{e}}-c\right|\right) /(k h)}{\sum_{k h=0.1}^{\Omega}\left(\left|c^{\mathrm{e}}\right|\right) /(k h)}\right. & \\
& \left.+\frac{\sum_{k h=0.1}^{\Omega}\left(\left|c_{\mathrm{g}}^{\mathrm{e}}-c_{\mathrm{g}}\right|\right) /(k h)}{\sum_{k h=0.1}^{\Omega}\left(\left|c_{\mathrm{g}}^{\mathrm{e}}\right|\right) /(k h)}+\frac{\sum_{k h=0.1}^{\Omega}\left(\left|A_{x}^{\mathrm{e}}-A_{x}\right|\right) /(k h)}{\sum_{k h=0.1}^{\Omega}\left(\left|A_{x}^{\mathrm{e}}\right|\right) /(k h)}\right),
\end{aligned}
$$

where $c^{\mathrm{e}}, c_{\mathrm{g}}^{\mathrm{e}}$ and $A_{x}^{\mathrm{e}}$ are the exact linear phase speed, group velocity and shoaling gradient, whereas $c, c_{\mathrm{g}}$ and $A_{x}$ are the approximate values taken from the two-layer model derived here. Note that errors in both $c$ and $c_{\mathrm{g}}$ are related to the accuracy of 
Table 1. $\alpha$-values from linear optimization

\begin{tabular}{ccccc}
\hline$\Omega(k h)$ & $\alpha_{1}$ & $\alpha_{2}$ & $\alpha_{3}$ & $\Delta_{\text {lin }}$ \\
\hline 3 & -0.092 & -0.256 & -0.625 & 0.002 \\
5 & -0.127 & -0.266 & -0.618 & 0.006 \\
10 & -0.128 & -0.262 & -0.618 & 0.02 \\
{$[4,4]$ Padé } & -0.248 & -0.459 & -0.741 & - \\
\hline
\end{tabular}

the two-layer dispersion relation. The right-hand side is divided by three, so as to normalize the total error created by the three different sources. All of the summations are divided by $k h$ so that errors at low wavenumbers are more important than high wavenumber errors. The reason for this weighting is a peculiarity of the optimization: it was possible to sacrifice low-wavenumber accuracy $(k h<1.5)$ for accuracy at higher wavenumbers. Accuracy at low wavenumbers is paramount for applications in shallow water, and hence the weighting. Summations are started at $k h=0.1$ also because of the $k h$ weighting, and the subsequent need to avoid division by zero. The upper summation limit, $k h=\Omega$, is determined such that the minimum $\Delta_{\text {lin }}$ is less than some threshold.

To test the linear properties of the model equations, $\Delta_{\text {lin }}$, which can be thought of as an overall relative error, will be set equal to three arbitrary values: 0.002, 0.006 and 0.020. The behaviour of the model equations at these error constraints will be scrutinized, and a 'proper' $\Delta_{\text {lin }}$ value will be recommended. A summary of the optimization results is shown in table 1 . Also shown in last row of the table are the $\alpha$-values required to create a $[4,4]$ Padé approximation using the two-layer dispersion relation. Note that with the $[4,4]$ Padé coefficients and $\Omega=5, \Delta_{\text {lin }}=0.2$, due to a poor shoaling description. Figure 2 shows the linear properties for the cases where $\Delta_{\text {lin }}=0.002$ and 0.006 . This figure also shows the dispersion properties corresponding to the $[4,4]$ Padé. The [4,4] Padé yields excellent phase-speed agreement up to $k h \approx 6$, good group velocity agreement to $k h \approx 3$, and an accurate shoaling gradient to only $k h \approx 1$. For the $\Delta_{\text {lin }}=0.002$ case, we can see that the linear dispersion properties (phase and group speed) are not quite as good as the [4,4] Padé approximation. Linear shoaling is reproduced very well up to $k h \approx 3$. Looking now at the $\Delta_{\text {lin }}=0.006$ case, the model phase speed has better deep-water accuracy than the $[4,4]$ Padé approximation. The price paid for this increased accuracy is a group velocity with a $c a .1 \%$ error for $k h>3$. The shoaling gradient, however, matches the linear solution quite well up to $k h \approx 9$, with a very good agreement to $k h \approx 5$. The results for the $\alpha$-values from $\Delta_{\text {lin }}=0.02$ are not plotted, due to the fact that they are nearly identical to the plotted $\Delta_{\text {lin }}=0.006$ values.

For the rest of the paper, all the results will employ the $\alpha$-values from the $\Delta_{\text {lin }}=$ 0.006 minimization. This set was chosen based on its overall good properties. It can be expected that phase and group velocity will be captured for $k h$-values up to 6 . Linear shoaling will be excellent up to a $k h \approx 5$, although still reasonable up to $k h \approx 9$.

The vertical profiles of the velocity components with the $\alpha$-values for $\Delta_{\text {lin }}=0.06$ are given in figure 3. Also plotted on this figure are the velocity profiles of Gobbi et al.'s (2000) high-order derivation, which is a one-layer model, including terms up to $O\left(\mu_{0}^{4}\right)$. In general, the profiles for the present model and Gobbi et al.'s results are 

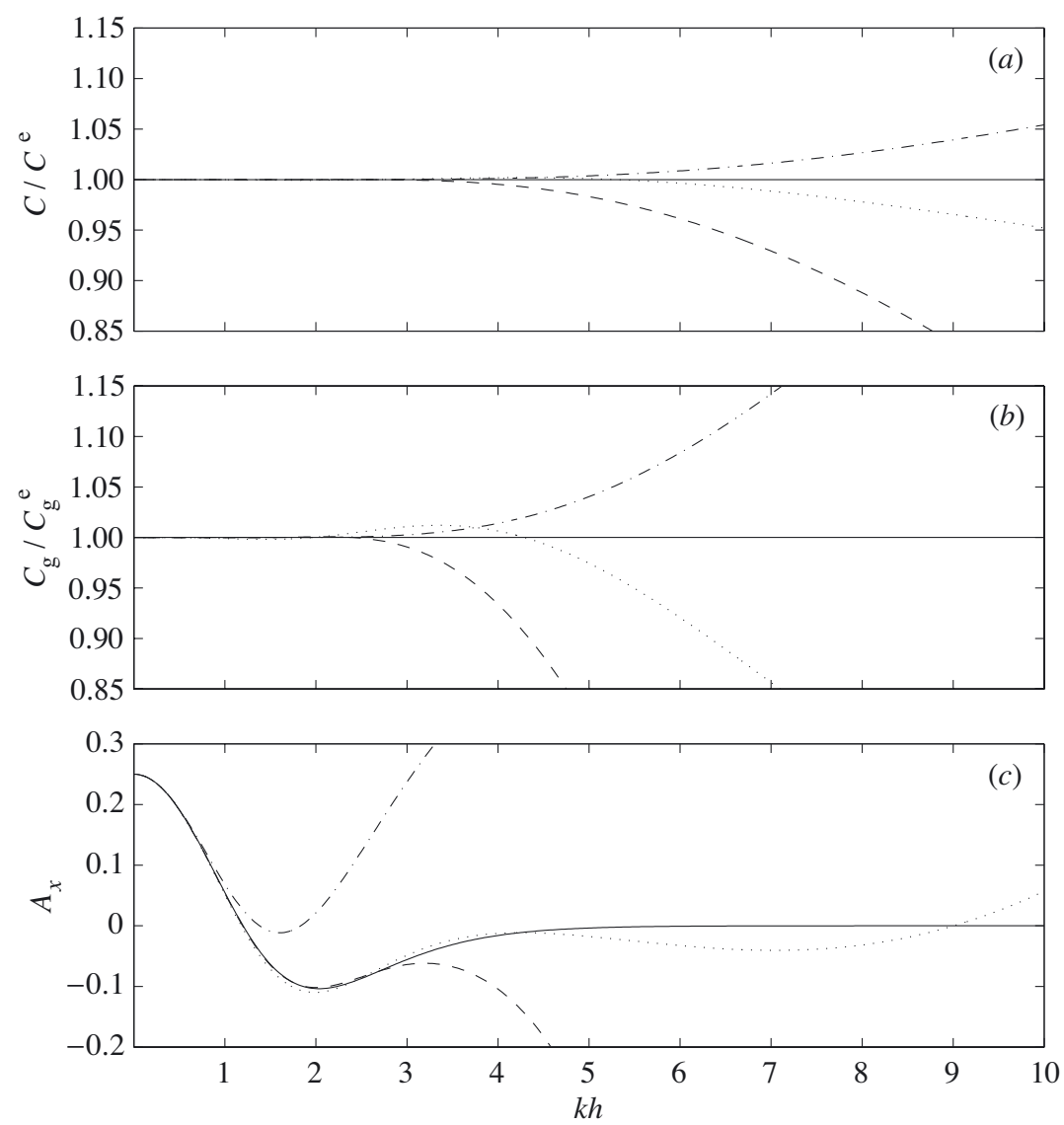

Figure 2. Linear properties of $(a)$ wave speed, $(b)$ group velocity and $(c)$ shoaling gradient of the two-layer model with $\alpha_{1}=-0.092, \alpha_{2}=-0.256, \alpha_{3}=-0.625\left(\Delta_{\text {lin }}=0.002\right)$ shown by the dashed line, and $\alpha_{1}=-0.127, \alpha_{2}=-0.266, \alpha_{3}=-0.618\left(\Delta_{\text {lin }}=0.006\right)$ shown by the dotted line. Comparison of wave speed and group velocity of the two-layer model with the exact linear relation (solid line); the dash-dotted line is the [4,4] Padé approximation.

similar, with the two-layer model exhibiting larger error in the case of $k h=3$ and significantly smaller error in the $k h=9$ case.

\section{(g) Choice of arbitrary levels: nonlinear optimization}

From the linear optimization of the previous section, the three levels are given as

$$
\kappa_{1}=-0.127 h+\beta_{1} \zeta, \quad \eta=-0.266 h+\beta_{2} \zeta, \quad \kappa_{2}=-0.618 h+\beta_{3} \zeta .
$$

In this section, through examination of nonlinear properties, the $\beta$ coefficients will be chosen. The nonlinear optimization detailed in this section is similar to that performed by Kennedy et al. (2001) while working with their one-layer model. Following the same procedure as the linear optimization, a representation of the nonlinear error, including errors in the second-order free-surface correction and subharmonic/superharmonic transfer functions is given by the minimization parame- 

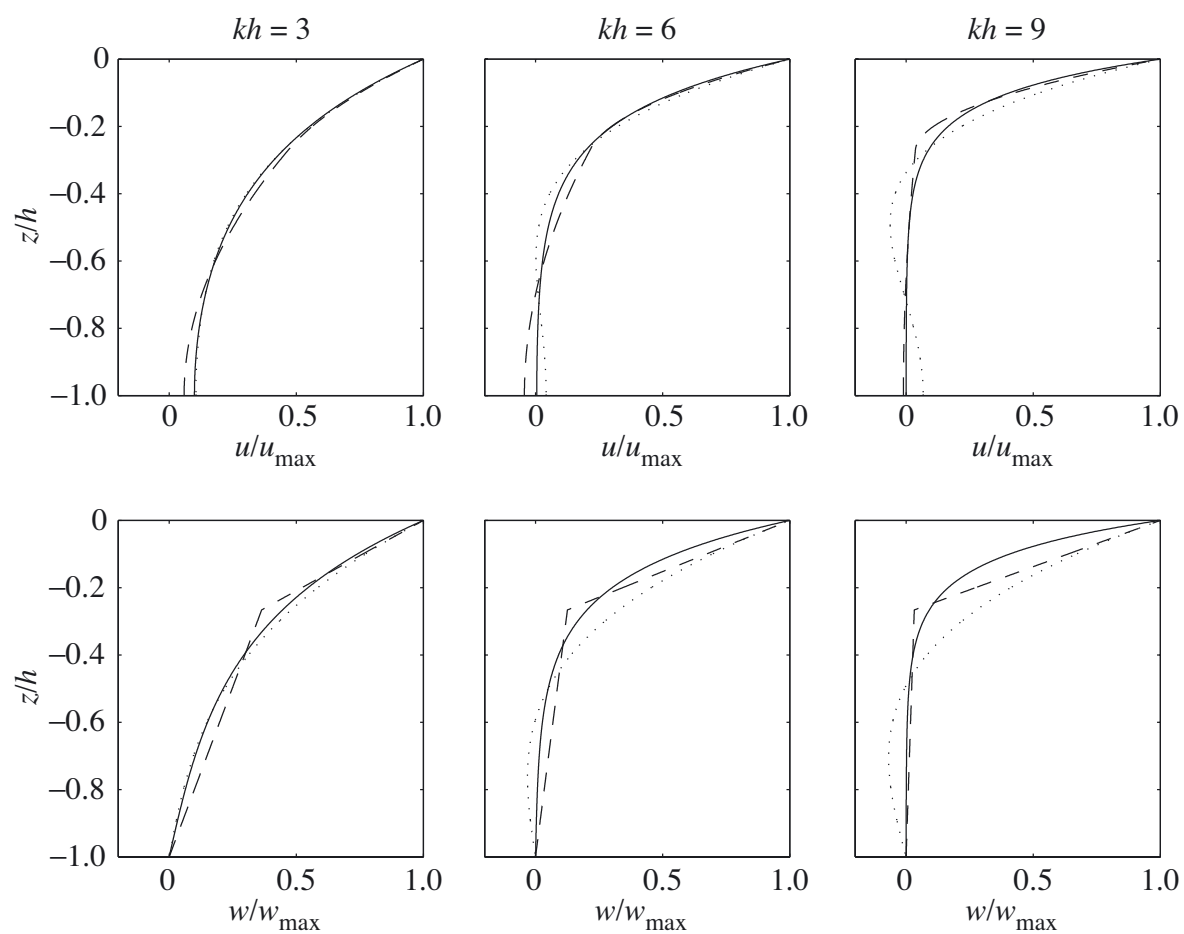

Figure 3. Vertical profile of horizontal velocity (top row) and vertical velocity (bottom row) under the crest of a sine wave for three different $k h$-values, as given by linear theory (solid line), the high-order model of Gobbi et al. (2000) (dotted line), and the two-layer model presented in this paper employing the $\Delta_{\text {lin }}=0.006$ coefficients (dashed line).

Table 2. $\beta$-values from nonlinear optimization

\begin{tabular}{rcrrrr}
\hline$\Omega(k h)$ & $\beta_{1}$ & \multicolumn{1}{c}{$\beta_{2}$} & \multicolumn{1}{c}{$\beta_{3}$} & \multicolumn{1}{c}{$\Delta_{\mathrm{NL}}$} \\
\hline 5 & 0.031 & -0.001 & -0.063 & 0.018 \\
10 & 0.74 & 1.037 & -0.234 & 0.034 \\
\hline
\end{tabular}

ter $\Delta_{\mathrm{NL}}$ :

$$
\begin{aligned}
\Delta_{\mathrm{NL}}=\frac{1}{2}\left(\frac{\sum_{k h=1}^{\Omega}\left(\left|\zeta_{\text {Stokes }}^{(1)}-\zeta^{(1)}\right|\right) /(k h)}{\sum_{k h=1}^{\Omega}\left(\left|\zeta_{\text {Stokes }}^{(1)}\right|\right) /(k h)}\right. & \\
& \left.+\frac{\sum_{k_{1} h=1}^{\Omega} \sum_{k_{2} h=1}^{\Omega}\left(\left|\zeta_{\text {Stokes }}^{\mp}-\zeta^{\mp}\right|\right) /\left(k_{1} h+k_{2} h\right)}{\sum_{k_{1} h=1}^{\Omega} \sum_{k_{2} h=1}^{\Omega}\left(\left|\zeta_{\text {Stokes }}^{\mp}\right|\right) /\left(k_{1} h+k_{2} h\right)}\right) .
\end{aligned}
$$

Note that the summation limit for the nonlinear error begins at $k h=1.0$. The extremely large values of these nonlinear parameters at $k h$-values less than one lead to poor error quantifications at higher wavenumbers when using this type of error formulation. Also, Stokes's wave theory yields a good physical description of wave propagation only for $k h$-values larger than one, and thus analysing and optimizing the model properties for $k h$-values less than one may not be important. Notice that 


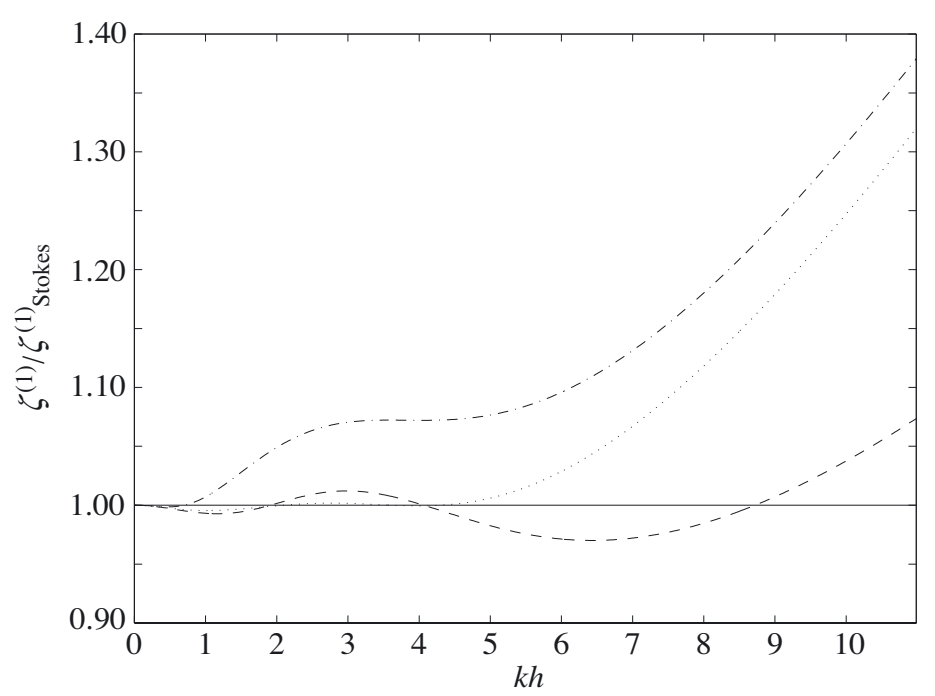

Figure 4. Second-order free-surface correction, $\zeta^{(1)}$, relative to the Stokes solution, with no nonlinear optimization $\left(\beta_{1}=\beta_{2}=\beta_{3}=0\right)$ shown by the dash-dotted line, the $\Delta_{\mathrm{NL}}=0.018$ results by the dotted line, and the $\Delta_{\mathrm{NL}}=0.034$ results by the dashed line.

the self-self interaction has an increased weighting in this error formulation, as it is included in both the terms comprising $\Delta_{\mathrm{NL}}$. A summary of the nonlinear optimization results is shown in table 2 . Figure 4 shows the second-order free-surface amplitude correction associated with these two sets of $\beta$, along with the correction without nonlinear optimization, i.e. $\beta_{1}=\beta_{2}=\beta_{3}=0$. The $\Delta_{\mathrm{NL}}=0.018$ shows excellent agreement to $k h \approx 7$, where the relative error is just over $5 \%$. After this point, the error grows quickly. For the $\Delta_{\mathrm{NL}}=0.034$ optimization, fairly large errors are found at $k h \approx 3$, although the error is much less for high $k h$ as compared with the $\Delta_{\mathrm{NL}}=0.018$ case. The bichromatic transfer amplitudes are shown in figure 5 , where the superharmonics are given in the upper left, and the subharmonics the lower right. For the case without nonlinear optimization, figure $5 a$ ), good agreement is only found at small $k h$-values for both super- and subharmonics. However, with some nonlinear optimization, as shown in figure $5 b$ ) for $\Delta_{\mathrm{NL}}=0.018$, the superharmonic amplitudes become much more accurate. In fact, transfers where $k_{1} h$ and $k_{2} h$ are close show excellent agreement, with the $5 \%$ error contour extending to $k_{1} h \approx k_{2} h \approx 6.5$. However, subharmonic transfer are relatively unaffected by the optimization, and lose accuracy quickly for $k_{1} h$-values greater than 4 . With respect to the subharmonic amplitudes, the same can be said for the $\Delta_{\mathrm{NL}}=0.034$ optimization as well, shown in figure $5 c$ ). Accurate superharmonics are better predicted for this optimization, where the $5 \%$ error contour extends to very deep water. It is noted that these transfer plots show very similar behaviour to those given by Kennedy et al. (2001) for the nonlinear-optimized one-layer model. In fact, the error of the two-layer model, for the $\Delta_{\mathrm{NL}}=0.018$ case, is approximately one-half of their one-layer model error at all $\left(k_{1} h, k_{2} h\right)$ combinations.

As with the linear optimization, choosing which set of $\beta$-values is best to use depends on the specifics of the problem to be examined. We choose to employ the 

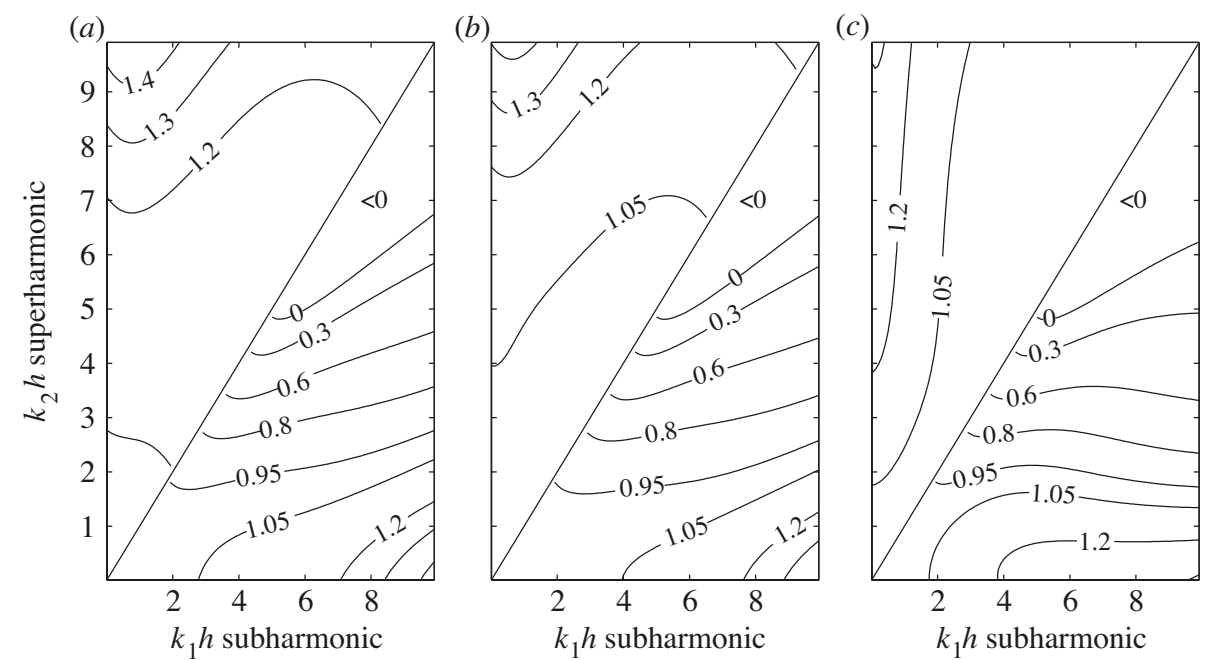

Figure 5. Sub- and superharmonic transfer amplitudes for bichromatic wave interactions, $\zeta^{\mp}$, relative to the Stokes solution, where subharmonics are shown in the lower right, and superharmonics in the upper left. (a) Results with no nonlinear optimization $\left(\beta_{1}=\beta_{2}=\beta_{3}=0\right)$, (b) $\Delta_{\mathrm{NL}}=0.018$ results, $(c) \Delta_{\mathrm{NL}}=0.034$ results.

set of coefficients from the $\Delta_{\mathrm{NL}}=0.018$ case. This set exhibits significantly better accuracy at all wavenumbers when $k h<6$, which is a highly desirable characteristic.

In this paper, nonlinear optimization is performed to second-order only. To optimize the model to third-order, for example, the best results would be achieved by continuation of the nonlinear expansion of the evaluation levels, i.e. the third-order expansion for the layer interface could take the form

$$
\eta=\alpha_{2} h+\beta_{2} \zeta+\frac{\left(\gamma_{2} \zeta\right)^{2}}{h}
$$

The coefficients $\gamma$ would determined such that an optimal agreement with third-order Stokes theory is obtained.

\section{Numerical solutions}

In this section some numerical solutions are obtained and compared with experimental data. The numerical solution procedure for the system of equations described in the previous section is first discussed here. All spatial derivatives are finite-differenced with centred differences. To keep the numerical truncation error consistent with the order of the model equations, all first-order spatial differences are differenced to fourth-order accuracy $\left(\Delta x^{4}, \Delta y^{4}\right)$, while second-order differences are taken to second-order accuracy. The continuity equation (2.34) is solved using the fourthorder predictor-corrector scheme as described in Wei et al. (1995) and Lynett \& Liu (2002), and will not be repeated here. The momentum equation (2.32) is also solved with the predictor-corrector approach. Examining the $x$-momentum equation, the 
$u_{1}$ time derivatives are grouped into a new variable $\mathcal{U}_{1}$, yielding

$$
\begin{gathered}
\frac{\partial \mathcal{U}_{1}}{\partial t}+\frac{1}{2} \frac{\partial u_{1}^{2}}{\partial x}+\frac{1}{2} \frac{\partial v_{1}^{2}}{\partial x}+g \frac{\partial \zeta}{\partial x} \\
+\frac{\partial}{\partial t}\left\{( \kappa _ { 1 } - \zeta ) \left[\eta\left(\frac{\partial^{2} u_{2}}{\partial x^{2}}+\frac{\partial^{2} v_{2}}{\partial x \partial y}-\frac{\partial^{2} v_{1}}{\partial x \partial y}\right)+\frac{\partial \eta}{\partial x}\left(\frac{\partial u_{2}}{\partial x}+\frac{\partial v_{2}}{\partial y}-\frac{\partial v_{1}}{\partial y}\right)\right.\right. \\
\left.+\frac{\partial^{2}\left(h u_{2}\right)}{\partial x^{2}}+\frac{\partial^{2}\left(h v_{2}\right)}{\partial x \partial y}+\frac{\partial^{2} h}{\partial x t}\right]+\frac{\kappa_{1}^{2}-\zeta^{2}}{2} \frac{\partial^{2} v_{1}}{\partial x \partial y}-\zeta \frac{\partial \zeta}{\partial x} \frac{\partial v_{1}}{\partial y} \\
\left.-\frac{\partial \zeta}{\partial x}\left[\eta\left(\frac{\partial u_{2}}{\partial x}+\frac{\partial v_{2}}{\partial y}-\frac{\partial v_{1}}{\partial y}\right)+\frac{\partial\left(h u_{2}\right)}{\partial x}+\frac{\partial\left(h v_{2}\right)}{\partial y}+\frac{\partial h}{\partial t}\right]\right\} \\
+\frac{\partial}{\partial x}\left[\frac{\partial \zeta}{\partial t}\left(\zeta S_{1}+T_{1}\right)+\frac{1}{2}\left(\kappa_{1}^{2}-\zeta^{2}\right)\left(u_{1} \frac{\partial S_{1}}{\partial x}+v_{1} \frac{\partial S_{1}}{\partial y}\right)\right. \\
\left.+\left(\kappa_{1}-\zeta\right)\left(u_{1} \frac{\partial T_{1}}{\partial x}+v_{1} \frac{\partial T_{1}}{\partial y}\right)+\frac{1}{2}\left(T_{1}+\zeta S_{1}\right)^{2}\right]=0
\end{gathered}
$$

where

$$
\mathcal{U}_{1}=u_{1}+\left[\frac{\kappa_{1}^{2}-2 \kappa_{1} \eta-\zeta^{2}+2 \zeta \eta}{2} \frac{\partial^{2} u_{1}}{\partial x^{2}}+\left(\eta \frac{\partial \zeta}{\partial x}+\zeta \frac{\partial \eta}{\partial x}-\kappa_{1} \frac{\partial \eta}{\partial x}-\zeta \frac{\partial \zeta}{\partial x}\right) \frac{\partial u_{1}}{\partial x}\right]=0 .
$$

This is the grouping procedure suggested by Wei et al. (1995) and modified to include nonlinear terms by Lynett \& Liu (2002). Equation (4.2) yields a diagonal matrix system after finite differencing, and can be solved efficiently. The lower-layer velocity is then calculated by

$$
\begin{aligned}
& u_{2}+\left\{\frac{\kappa_{2}^{2}+\eta^{2}-2 \eta \kappa_{1}}{2} \frac{\partial^{2} u_{2}}{\partial x^{2}}+\left(\kappa_{2}-\kappa_{1}\right) \frac{\partial^{2}\left(h u_{2}\right)}{\partial x^{2}}+\left(\eta-\kappa_{1}\right) \frac{\partial \eta}{\partial x} \frac{\partial u_{2}}{\partial x}\right\} \\
&=u_{1}+\left\{\frac{\left(\kappa_{1}-\eta\right)^{2}}{2}\left(\frac{\partial^{2} u_{1}}{\partial x^{2}}+\frac{\partial^{2} v_{1}}{\partial x \partial y}\right)+\left(\kappa_{1}-\eta\right)\left[\frac{\partial \eta}{\partial x}\left(\frac{\partial v_{2}}{\partial y}-\frac{\partial u_{1}}{\partial x}-\frac{\partial v_{1}}{\partial y}\right)\right]\right. \\
&\left.\quad+\frac{2 \eta \kappa_{1}-\kappa_{2}^{2}-\eta^{2}}{2} \frac{\partial^{2} v_{2}}{\partial x \partial y}+\left(\kappa_{1}-\kappa_{2}\right)\left[\frac{\partial^{2}\left(h v_{2}\right)}{\partial x \partial y}+\frac{\partial^{2} h}{\partial x t}\right]\right\},
\end{aligned}
$$

which is also diagonal, and is solved immediately after (4.2). Similar equations can be derived for the $v$ velocity components. No filtering or other artificial numerical dissipation is used. As the equation form of the presented two-layer model is identical to the $O\left(\mu_{0}^{2}\right)$ Boussinesq-type equations, the numerical details, such as convergence criteria, are identical to those given in Wei et al. (1995) and Lynett \& Liu (2002). For all of the numerical simulations presented in this paper, the lateral boundaries are modelled as absorbing boundaries through the use of sponge layers. The sponge layers are applied for both the one- and two-layer models as recommended by Kirby et al. (1998) for their one-layer model, which is an approach founded on the method presented by Israeli \& Orszag (1981).

In general, the two-layer model requires twice the computational time of an identically configured one-layer simulation. Through one iteration of the predictor and corrector procedure, the two-layer model needs about 1.75 times as much computational time as a one-layer model does. Due to the increased number of unknowns, the 
two-layer model takes 1.0-1.25 times as many iterations than the one-layer model to converge. With the combination of these two factors, the doubled computational time requirement is explained.

\section{(a) Highly nonlinear solitary waves}

As a first application of the two-layer model, the properties of a very large amplitude solitary wave are analysed. The solitary wave represents a balance of nonlinear and dispersive effects, thereby creating a wave of permanent form. When the amplitude of a solitary wave is small, the characteristic length of the wave is relatively large, and weakly nonlinear, weakly dispersive theories can describe the soliton very well (e.g. Wei \& Kirby 1995). However, as the amplitude increases, the wavelength shortens, and therefore higher-order nonlinear and dispersive effects become more important. This phenomenon is discussed in detail in Gobbi et al. (2000), where it was shown that models with nonlinear accuracy into the deep-water regime are required to accurately simulate highly nonlinear solitary waves. One of the examples presented in their paper will be investigated again here, with the two-layer model results included.

As an initial condition for the numerical model, the solitary-wave solution of Wei \& Kirby (1995), which is derived from the weakly nonlinear one-layer model, is inputted into the domain. As this wave solution is not the solitary-wave solution of the twolayer model, when the numerical simulation is started the soliton 'sheds' some waves, as a tail. The soliton, which moves rapidly due to its large amplitude, eventually leaves this tail far behind and reaches a steady form. Due to this initial fluctuation in wave form, the amplitude of the initial condition is chosen based on trial and error until a solitary wave with the desired amplitude is generated. This initial transient state is also mentioned in Gobbi et al. (2000). For the solitary wave examined in this section, with an amplitude of $0.65 h$, roughly 100 water depths of propagation were needed to separate the solitary wave from its trailing tail. Additionally, for this simulation, a grid length, $\Delta x$, of $0.04 h$ and a Courant number $(\Delta t \sqrt{g h}) / \Delta x$ of 0.5 , where $\Delta t$ is the time-step were employed. The numerical solitary wave of the two-layer model will be compared with the numerical waveform of the one-layer model, the high-order model of Gobbi et al. (2000), and the exact solution to the full boundary-value problem presented by Tanaka (1986).

Figure 6 shows a comparison of the free-surface profiles of the four different solutions. The two-layer model matches the exact solution to a very high accuracy, although there is some small error for $x / h>2$, which is difficult to discern in the figure. The two-layer model shows the best agreement with the exact solution as compared with the one-layer model and the high-order model of Gobbi et al. (2000). In figure 7 , the vertical profiles of horizontal velocity under the wave crest for the various models are shown. Near the seafloor, the two-layer model exhibits the best agreement with the exact solution, although the difference between the two-layer model and Gobbi et al.'s high-order model is small. Moving towards the free surface, these two models converge in their predictions, showing equal errors in the maximum horizontal velocity. It is clear from these comparisons that the two-layer model captures to a highly accurate degree the physics of a nonlinear solitary wave, with agreement on a par with or slightly better than that found with the model of Gobbi et al. (2000).

Proc. R. Soc. Lond. A (2004) 


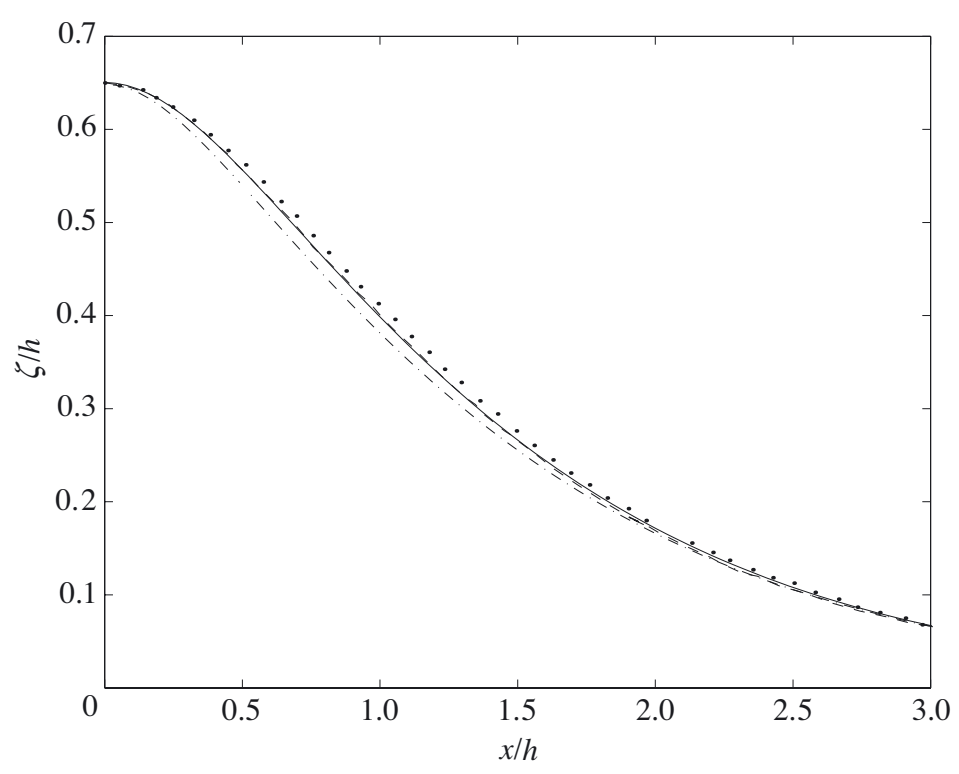

Figure 6. Shape of solitary wave with amplitude $\zeta / h=0.65$. The exact solution is given by the dashed line, the two-layer model by the solid line, the Gobbi et al. (2000) high-order model by the dots, and the one-layer model by the dash-dotted line.

\section{(b) Wave propagation over a submerged bar}

In this section, wave interaction with a submerged bar is examined. The setup is taken from the experiments presented by Dingemans (1994), who recorded free-surface time-series at numerous locations in front of and behind the obstacle. The orientation of the bar is shown in the top subplot of figure 8. The wave, as it approaches the bar, is a long wave, with a $k h=0.7$ (wavelength of $7.7 \mathrm{~m}$ in $0.86 \mathrm{~m}$ of water). This incident wave corresponds to Case A in Dingemans (1994). As the wave shoals, it steepens and nonlinear transfers create superharmonics. The superharmonics, while still shallow or intermediate water waves on top of the bar, become deep-water waves as they enter the deeper water behind. As discussed in Woo \& Liu (2001), significant wave energy (ca.75\% of the peak spectral amplitude) is present at $k h \approx 4$ in the region behind the bar. For this reason, Boussinesqtype models (one-layer $O\left(\mu_{0}^{2}\right)$ models), whose linear dispersion accuracy limit is near $k h \approx 3$, do not correctly predict the wavefield behind the bar.

The numerical simulation results for this case are shown in figure 8 , where $\Delta x=$ $0.10 \mathrm{~m}$ and $\Delta t=0.0189 \mathrm{~s}$. Time-series are taken at the four locations depicted in the top subplot, and both the one- and two-layer models are compared with experimental data. The column on the left shows the one-layer results and the column on the right shows the two-layer results. On top of the bar, at location \#1, both models are in agreement, and the two-layer model shows no benefit. This is expected, as all of the dominant wave components at this location have $k h$-values less than 2.0. However as the wave components progress into deeper water, the one-layer model becomes inaccurate. This is evident at locations \#2-\#4, where the one-layer model deviates from the experimental results. The two-layer model, on the other hand, shows its strength and predicts the wavefield excellently. 


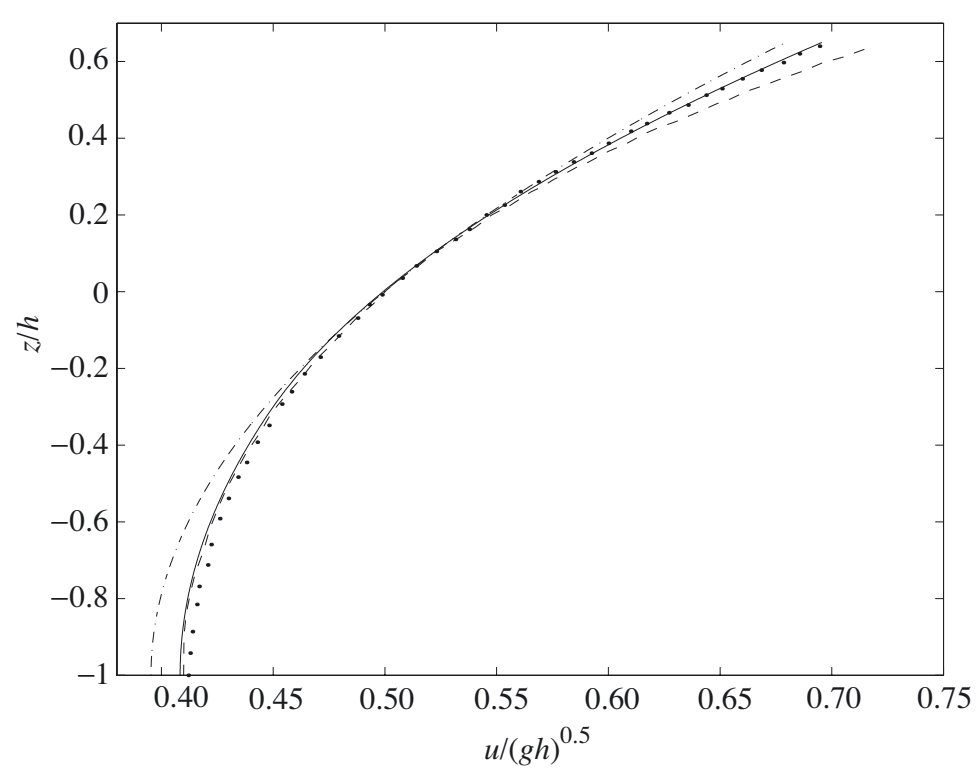

Figure 7. Vertical profile of horizontal velocity under the crest of a solitary wave with amplitude $\zeta / h=0.65$. The exact solution is given by the dashed line, the two-layer model by the solid line, the Gobbi et al. (2000) high-order model by the dots, and the one-layer model by the dash-dotted line.

A second case was presented in Dingemans (1994), that for a shorter incident wave travelling over the same submerged bar as discussed above. This experimental dataset, named Case C in Dingemans (1994), employs an intermediate depth wave with a $k h=1.8$ (wavelength of $3.0 \mathrm{~m}$ in $0.86 \mathrm{~m}$ of water). As the wave passes over the top of the bar, energy is transferred into the second harmonic, with only a small amount of energy in the third harmonic behind the step. The second harmonic has a $k h=6.3$ in the $80 \mathrm{~cm}$ water depth behind the step, and certainly it is not expected that the one-layer model should correctly predict the wavefield in this region. Figure 9 proves this expectation, while the two-layer model captures the free surface extremely well. This particular case also exhibits the strength of the two-layer model over high-order Boussinesq-type models with dispersion properties equivalent to a $[4,4]$ Padé approximation of the dispersion relation. For the $k h=6.3$ component behind the step, the [4,4] Padé approximation predicts a celerity $c a .1 \%$ faster than that given by linear potential theory. Between locations \#3 and \#4 in figure 9 , this $k h=6.3$ wave will have travelled more than five wavelengths, equating to a minor arrival-time error in the vicinity of $5 \%$. These phase errors are evident in the numerical results given by Gobbi \& Kirby (1999), who compared a high-order model $([4,4]$ Padé representation of the dispersion relation) with experimental data very similar to that given in Dingemans (1994) and compared here. As the two-layer model presented in this paper has a $0.1 \%$ celerity error at $k h=6.3$, there are no noticeable phase differences in figure 9 .

\section{(c) Wave generation by submarine landslide}

The derivation presented in this paper did not make the simplifying assumption of a constant seafloor elevation in time. This will allow for the study of waves generated 

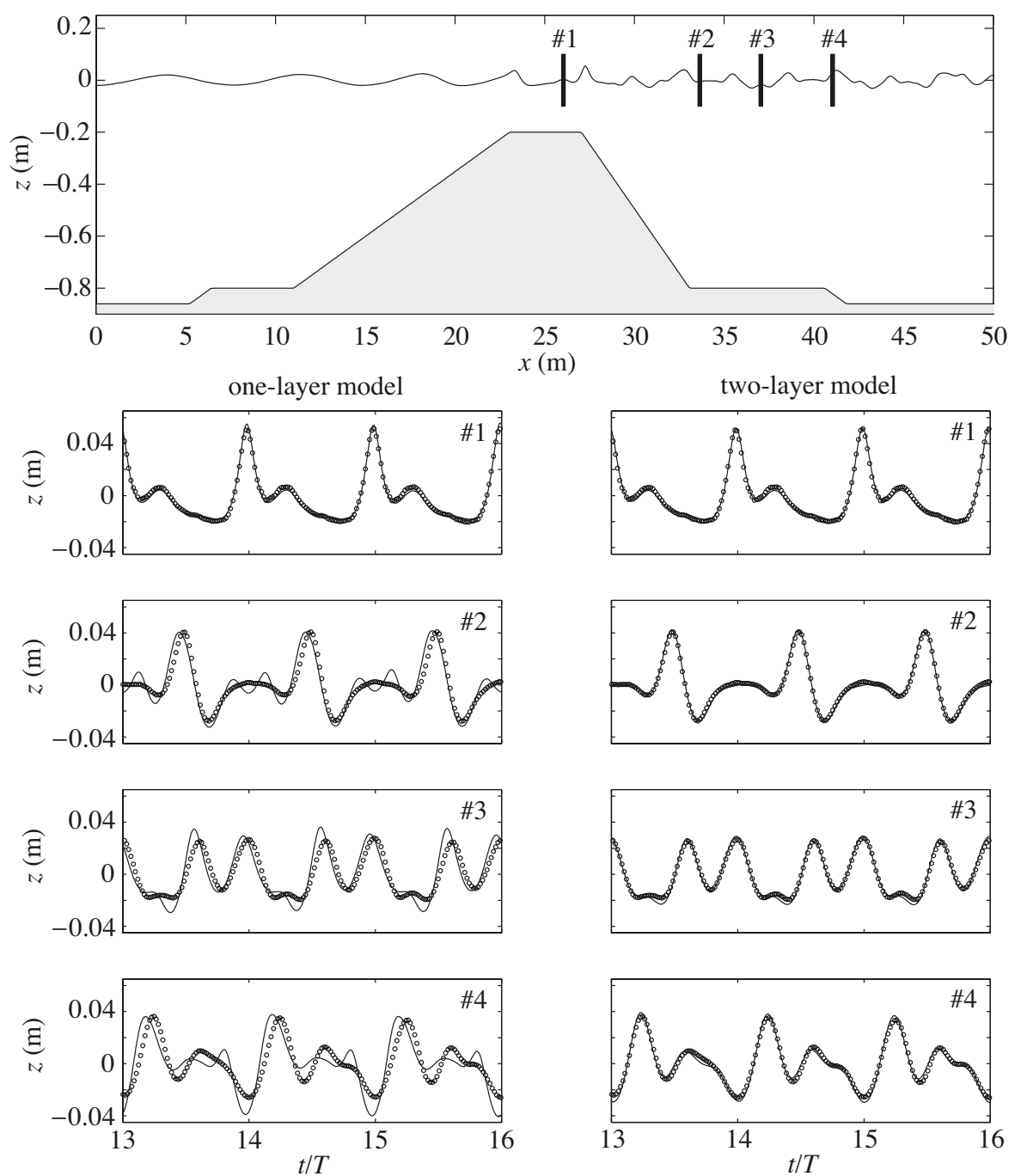

Figure 8. Comparison between numerical (solid lines) and experimental (dots) free-surface displacements for Case A of Dingemans (1994), where the experimental set-up and gauge locations are shown in the top subplot. The column on the left shows the numerical results from the one-layer model, the right column shows the two-layer results. Time-series locations are indicated in the upper right of each subplot, corresponding to the gauge locations shown in the top subplot.

by seafloor movements, such as submarine landslides and slumps. Using the onelayer-model equations to study wave creation by submarine seafloor movements has been examined previously by the authors (Lynett \& Liu 2002). In that work, a deepwater accuracy limitation, in regard to accurate prediction of the generated waves, of the one-layer model was determined. Focusing on a submarine slide mass that is symmetric about its centre point in the horizontal plane, a single length-scale of the slide mass can be given, $l_{\mathrm{s}}$, the horizontal length of the slide. For the onelayer model, accuracy is expected as long as $l_{\mathrm{s}} / h_{\mathrm{c}}>7$, where $h_{\mathrm{c}}$ is the water depth 

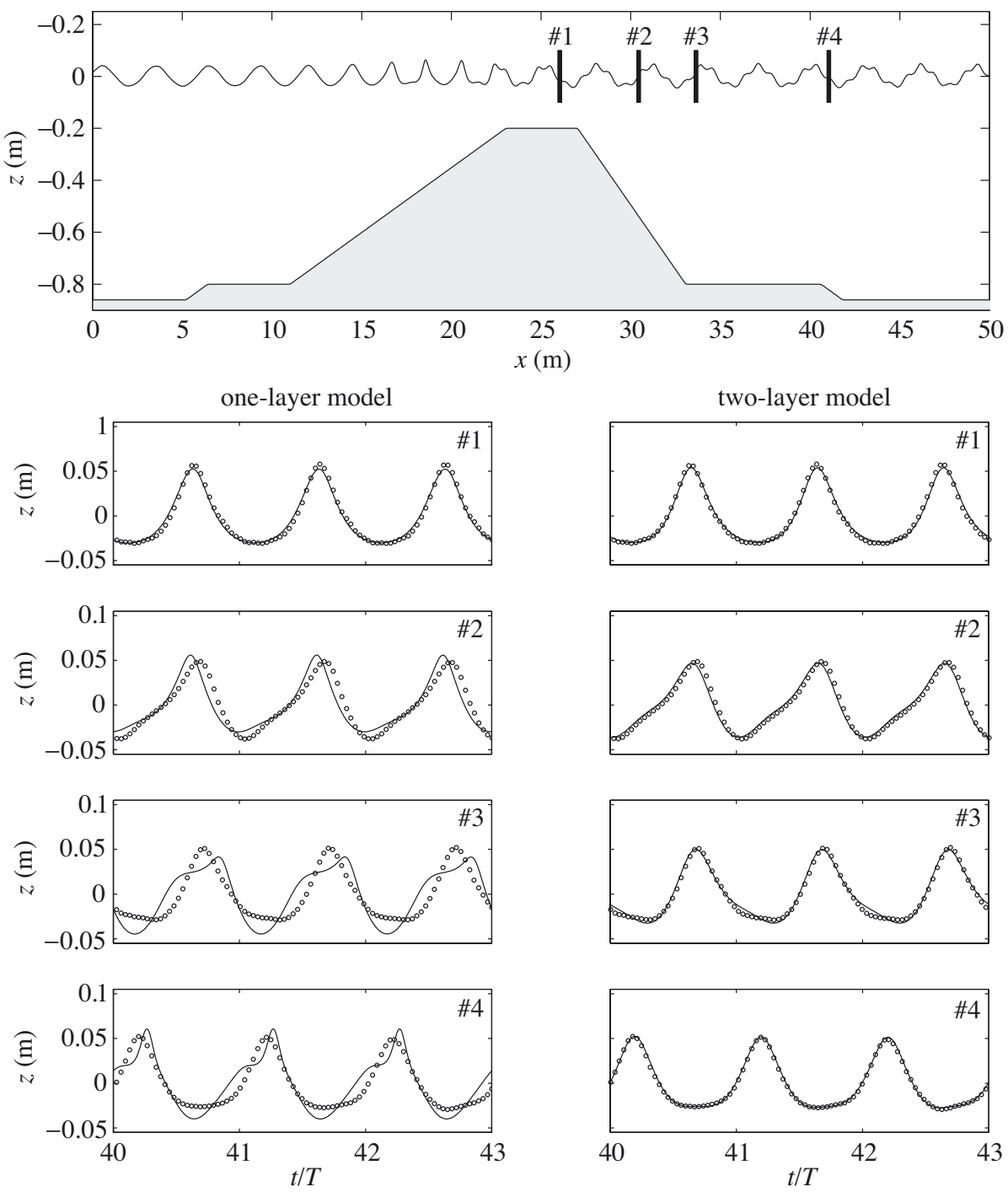

Figure 9. Comparison for Case C of Dingemans (1994), where the figure notation is as in figure 8 .

above the centre point of the slide mass. This accuracy limitation was found through comparison of the one-layer model with a fully nonlinear potential flow calculation. One of the comparisons presented in the paper by Lynett \& Liu (2002) (specifically, $\S 7$, 'Limitations of the depth-integrated model') is re-examined here with the present two-layer model.

The scenario to be recreated in this section involves a non-deforming mass translating down a planar slope in solid body motion. The mathematical description of the slide evolution can be found in Lynett \& Liu (2002, fig. 4) and will not be repeated here. This particular problem, already simulated using potential flow theory and a one-layer model, is calculated again with the two-layer equations, and the results are shown in figure 10. The top subplot of this figure shows the location of the slide mass 

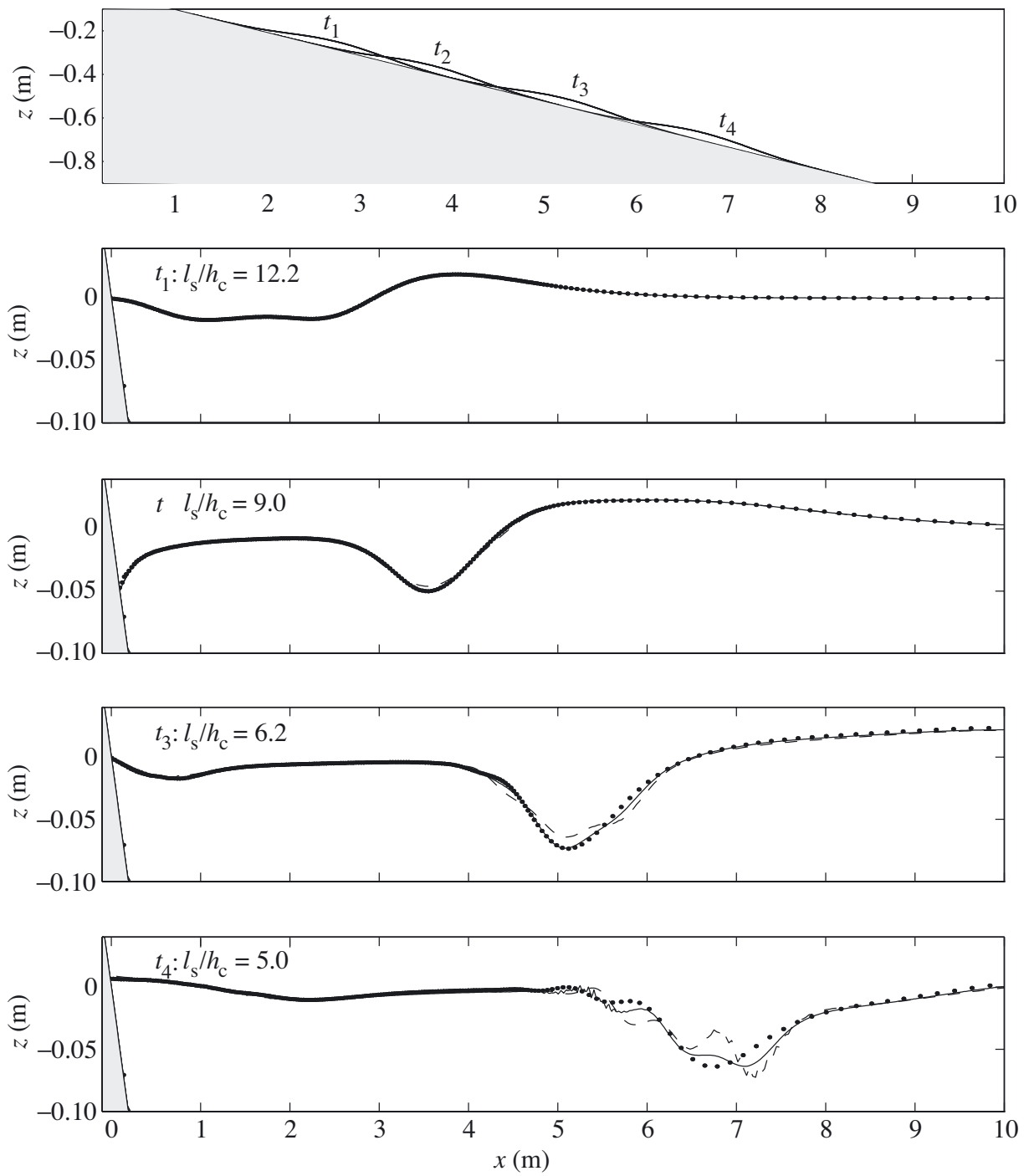

Figure 10. Numerical simulation of a submarine landslide. The top plot shows the location of the slide mass at the four times corresponding to the free-surface snapshots in the four lower plots. Free-surface snapshots for potential flow theory (dots), one-layer model (dashed line), and two-layer model (solid line) are given.

at four different times, which correspond to the free-surface snapshots shown in the lower four subplots. Given on each of these four plots is the $l_{\mathrm{s}} / h_{\mathrm{c}}$ ratio at the time the snapshot is taken. Note that $l_{\mathrm{S}}$ is constant in time, but as the slide moves into deeper water, $h_{\mathrm{c}}$ increases. For the times $t_{1}$ and $t_{2}$, all three models agree, and the depth-integrated models are still in the range of accuracy. As can be seen by time $t_{3}$, the one-layer model begins to diverge from the potential flow results, indicating that the slide is in water too deep for this model to handle accurately. At this time, the two-layer model is still in excellent agreement with potential theory. By time $t_{4}$, the two-layer model is beginning to differ from potential theory. Although the rigorous 


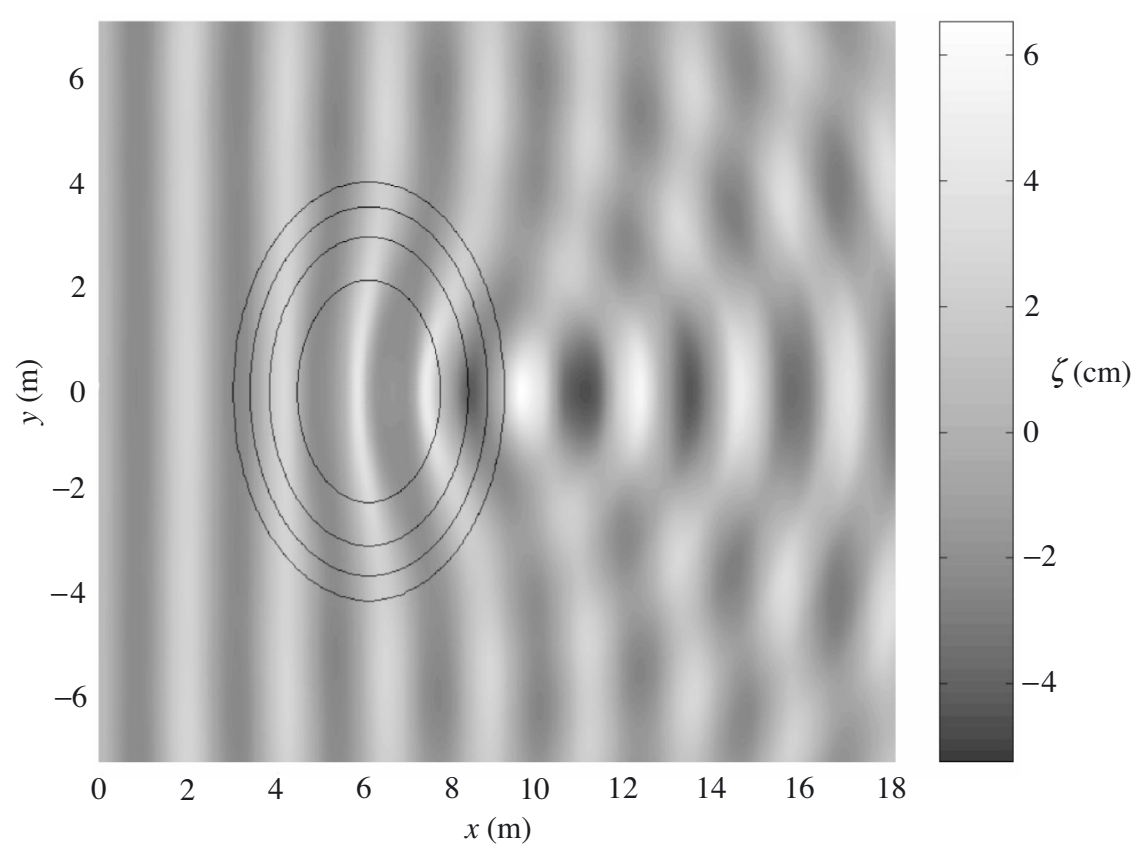

Figure 11. Numerical snapshot from a two-layer, 2HD shoal simulation, where the location of the shoal is denoted by the dashed contours. The snapshot is taken $32 \mathrm{~s}$ into the simulation, or roughly 24.6 wave periods.

determination of the accuracy limit for the one-layer model made in Lynett \& Liu will not be repeated here for the two-layer model, it is clear that the two-layer model gives more accurate results into deeper water than the one-layer model does.

\section{(d) 2HD wave evolution over a shoal}

One of the most frequently studied 2HD problems is that of wave interaction with a submerged elliptic shoal. Experiments by Berkoff et al. (1982), Vincent \& Briggs (1989) and others have been used repeatedly to validate mild-slope equations models (e.g. Liu et al. 1985) as well as Boussinesq-type models (e.g. Chen et al. 2000). The submerged shoal is a particularly desirable $2 \mathrm{HD}$ validation problem because the wavefield behind the shoal can vary greatly in both the along-channel and crosschannel directions, indicating that the $2 \mathrm{HD}$ effects are very important.

In this paper, one of the experiments of Vincent \& Briggs (1989) will be numerically simulated. The elliptic shoal is $6.1 \mathrm{~m}$ long in the $x$-direction and $7.92 \mathrm{~m}$ wide in the $y$-direction, with a maximum height of $30.5 \mathrm{~cm}$ in $45.7 \mathrm{~cm}$ of water. The precise mathematical description of the shoal can be found in Vincent \& Briggs. A large variety of incident wave conditions was studied, ranging from non-breaking monochromatic waves to breaking directional spectra. A non-breaking monochromatic incident condition is examined in this paper. The incident wave has a height of $4.8 \mathrm{~cm}$ and a period of $1.3 \mathrm{~s}(k h=1.27)$. The incident wave height was chosen based on agreement with experimental data taken nearest to the wavemaker, which varied significantly from the experimental target wave height of $5.5 \mathrm{~cm}$. The numerical parameters of the simulations are $\Delta x=0.056 \mathrm{~m}$ and $\Delta t=0.026 \mathrm{~s}$. A snapshot of the quasi-steady state 


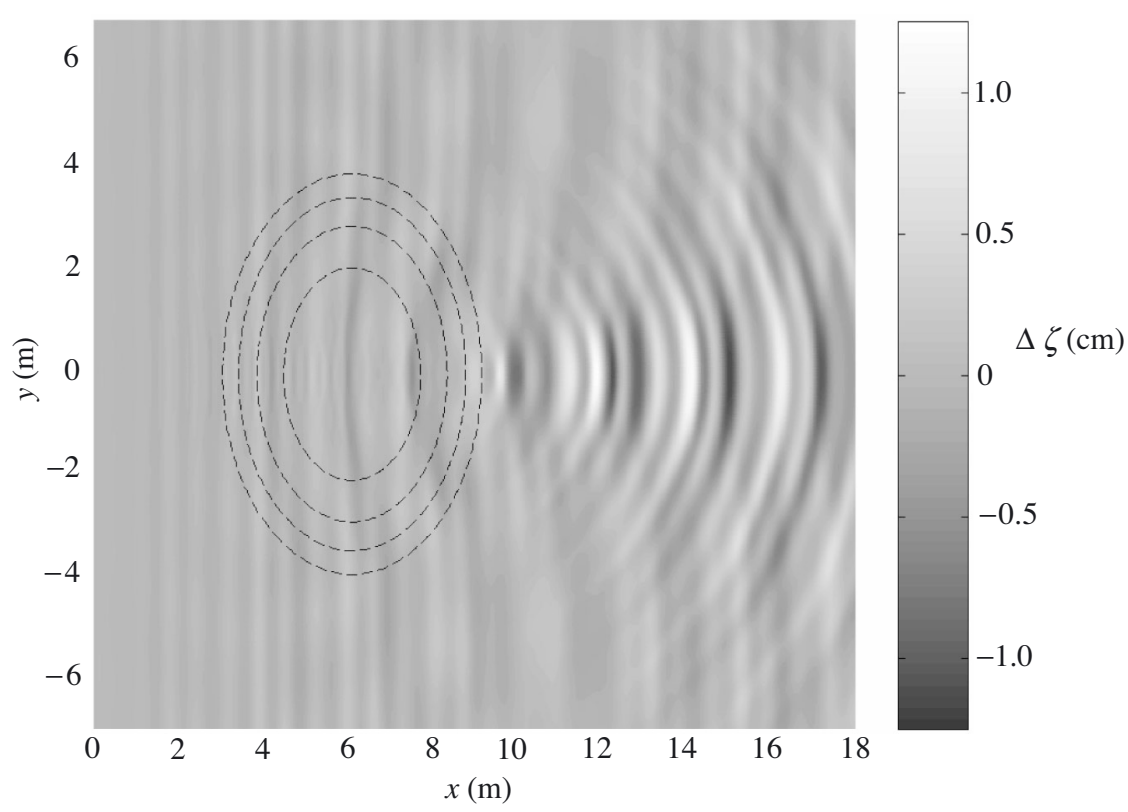

Figure 12. Difference between one- and two-layer numerical simulations at a time of $32 \mathrm{~s}$. The location of the shoal is denoted by the dashed contours.

free surface, taken $32 \mathrm{~s}$ into the simulation, is shown in figure 11. From this image, the processes of wave transformation can be explained. The waves approach from the left and, in passing over the shoal, the wavefront slows in the shallower water, and the wave crest narrows and steepens. On the lee of the shoal, refracting wavefronts meet, creating a free-surface elevation maximum. Oblique wave interactions dominate the wavefield behind the shoal, creating an irregular sea surface.

Both one- and two-layer simulations were performed, and the difference in the free-surface elevation predicted by the models at a time of $32 \mathrm{~s}$ is shown in figure 12 . This instantaneous difference shows that the wavefields predicted by the models behind the shoal do not agree particularly well. Differences in the models are in the range of $\mp 1.25 \mathrm{~cm}$, in an area where the wave has an amplitude of $4-5 \mathrm{~cm}$. As in the one-horizontal-dimension problem of $\S 4 a$, it is expected that the higher-frequency waves generated due to nonlinear transfers on the shoal are not predicted accurately by the one-layer model behind the shoal. Figure 13 proves this expectation. First, however, given in figure 13b) is the experimental wave height along the centreline of the channel $(y=0)$ plotted with the one- and two-layer values. The trends of the experimental and numerical data are very similar, with small but clear differences between the one- and two-layer models behind the shoal.

Parts $(c)$ and $(d)$ of figures 13 are the first and second harmonic amplitudes, respectively, from the one- and two-layer simulations. The two models agree closely on first harmonic amplitudes, but do exhibit $0.25 \mathrm{~cm}$ differences behind the shoal. Figure $13 c$ shows very clearly the focusing of wave energy behind the shoal. The refracting wavefronts meet and create a peak height near $x=10 \mathrm{~m}$, after which the wave energy spreads laterally, decreasing the first harmonic amplitude. Looking at the second harmonic amplitudes, the results of the two models diverge quickly behind 

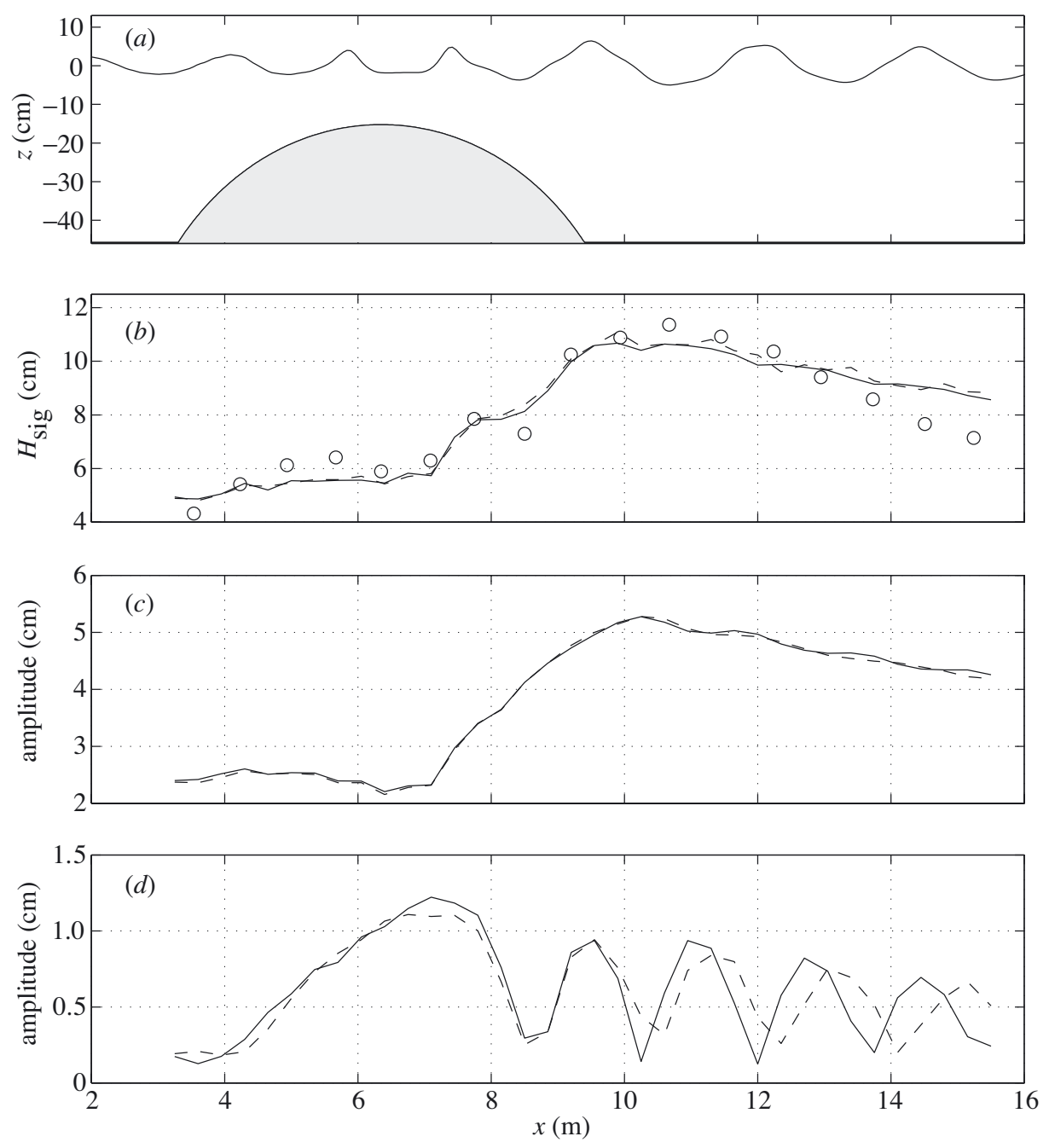

Figure 13. Characteristics of the elliptic shoal case, along the channel centreline $(y=0)$. $(a)$ The centreline depth profile. (b) The root-mean-square wave height of the Vincent \& Briggs (1989) experiments (dot-dashed line, where dots represent measurement locations), one-layer model results (dashed line), and two-layer model (solid line). (c) First and (d) second harmonic amplitudes, respectively, for the one- and two-layer models.

the shoal. In the deep water behind the shoal, this second harmonic has $k h=4.35$, and therefore the one-layer model will not be able to capture properly the physical properties of this wave component. This fact also explains the differences shown in figure 12, where the errors in the one-layer model are directly attributable to the incorrect phase-speed prediction of the second harmonic. Therefore, for this type of problem, the accuracy of the one-layer model correlates directly to the magnitude of energy transfer into the second harmonic. The two-layer model will capture the second harmonic properly, although if energy transfer into the third harmonic $(k h=$ 9.8 in the water behind the shoal) is significant, even this model will have errors.

Proc. R. Soc. Lond. A (2004) 
Table 3. Comparison of different aspects of the high-order models of Schäffer $\mathscr{G}$ Madsen (1995) with truncation error $\left[O\left(\epsilon_{0} \mu_{0}^{2}, \mu_{0}^{4}\right)\right]$

(A, flat bottom equations of Gobbi et al. (2000) $\left[O\left(\mu_{0}^{6}\right)\right]$; B, Madsen et al. $(2002)\left[O\left(\mu_{0}^{10}\right)\right]$; C, Madsen et al. (2003) $\left[O\left(\mu_{0}^{6}\right)\right]$; D, the two-layer model presented here $\left.\left[O\left(\mu_{0}^{2} \mu_{1}^{2}, \mu_{2}^{4}\right)\right].\right)$

\begin{tabular}{ccc}
\hline model & $\begin{array}{c}k h \text { at } 1 \% \\
\text { error in } c\end{array}$ & $\begin{array}{c}k h \text { at } 5 \% \\
\text { error in } \zeta^{(1)}\end{array}$ \\
\hline Schäffer \& Madsen (1995) & 6 & $1.2^{\text {a }}$ \\
A & 6 & 3.5 \\
B & 40 & 35 \\
C & 10 & 10 \\
D & 6.5 & 6.5 \\
\hline model & no. of eqns & no. of gradient operations \\
[no. of vector eqns] & $>3$ rd order] \\
\hline Schäffer \& Madsen (1995) & $2[1]$ & $41[0]^{\text {a }}$ \\
A & $2[1]$ & $42[5]$ \\
B & $6[3]$ & $98[12]$ \\
D & $6[3]$ & $44[0]$ \\
& $3[2]$ & $42[0]$ \\
\hline
\end{tabular}

${ }^{\mathrm{a}}$ Data taken from Madsen \& Schäffer (1998) $\left[O\left(\epsilon_{0}^{2} \mu_{0}^{2}, \mu_{0}^{4}\right)\right]$.

The amplitude of the third harmonic for the case analysed above is less than $0.2 \mathrm{~cm}$ in the region behind the shoal.

\section{Comparisons with existing high-order approaches}

As there are a number of high-order, Boussinesq-like approaches in the published literature, it is important to discuss how this model compares. A brief overview of the existing models is given now. One of the first high-order derivations was that of Schäffer \& Madsen (1995), who combined the operator manipulation method (Madsen \& Sørensen 1992) with Nwogu's (1993) derivation to create a two-equation model (mass and vector momentum), while only containing third-order spatial derivatives. A limitation of this approach is that, due to the operator manipulation, a velocity profile consistent with the final equations is not given. Continuing along a similar path, Gobbi et al. (2000), expanded Nwogu's approach to the next order, creating a two-equation model with fifth-order spatial derivatives. A novel approach to Boussinesq models was presented by Agnon et al. (1999), whereby the boundary conditions are satisfied exactly, and the Laplace equation is solved through expansions. This model, extended and enhanced in Madsen et al. (2002), solves six equations (three vector equations) and distinguishes itself from other models in its extremely accurate deep-water, nonlinear behaviour. Madsen et al. (2003) further analysed this approach, and described a version including only third-order spatial derivatives.

In an attempt to discriminate the advantages and disadvantages of these models as well as the two-layer model, four items are compared: $k h$ at $1 \%$ error in phase speed, $k h$ at $5 \%$ error in the self-self, second-order nonlinear (superharmonic) interaction, number of equations, and total number of gradient operations in the equations. These 
items, given in table 3 , are meant to yield a very simple comparison of the theoretical and practical aspects of different high-order models.

All of the values shown in table 3 are taken directly from the corresponding papers. For Madsen et al. (2002), results given above are for their $\sigma=-0.2$ and eqns (2), (15), (17) and (22b). For Madsen et al. (2003), results given above are for $\sigma=-0.5$, given in table 2 in their appendix. The equations for Madsen et al. (2003) are similar to Madsen et al. (2002), except with all derivatives higher than third-order truncated. Note that, when counting gradient operations, a first-order derivative counts as one, for example, and a fifth-order derivative counts as five. This count is performed on the final model equations, as presented by each author. Of course, many of the derivatives are repetitive, and would be stored in memory rather than recalculated in a numerical model; thus one should not look too deeply into the meaning of these numbers. The number of vector equations and spatial gradients greater than thirdorder are included in brackets as an indication of additional work for $2 \mathrm{HD}$ problems, as well as the numerical stencil size. Evident from table 3 is that accuracy is closely related to required effort, although the trend is not necessarily linear. The approaches of Madsen et al. have linear and first-order nonlinear accuracy into the deepest water, as well as the best second-order nonlinear accuracy, not shown here. The models of Gobbi et al. (2000) and Madsen et al. (2002) are likely to require significant additional effort to go from $1 \mathrm{HD}$ to $2 \mathrm{HD}$, while the computational requirements of Madsen et al. (2003) and the two-layer model would possibly be very similar. Naturally, the method of numerical solving can greatly affect these judgements.

\section{Conclusions}

A model for the transformation of highly nonlinear and dispersive waves is derived. The model uses two quadratic polynomials to approximate the vertical flow field, matched along an interface. Through linear and nonlinear optimization of the interface and velocity evaluation locations, it is shown that the two-layer model exhibits accurate linear and nonlinear characteristics up to $k h \approx 6.5$. This is an extension to higher $k h$ over existing $O\left(\mu_{0}^{4}\right)$ truncation error Boussinesq-type models, while maintaining the maximum order of differentiation at three. Owing to this maximum order of differentiation, a tractable numerical algorithm is developed for the general $2 \mathrm{HD}$ problem, employing a well-studied predictor-corrector scheme. A number of 1HD and 2HD simulations are given, and the presented model shows its strength by accurately capturing the propagation of highly nonlinear and deep-water waves. The ellipticshoal simulations presented represent the first attempt at high-order modelling of a $2 \mathrm{HD}$ problem.

It is possible that, for certain situations, the two-layer model would not be adequate in regard to simulating a correct picture of the wavefield. One example of this would be for the 2HD-shoal problem discussed in $\S 4 d$. If the incident wave had a greater amplitude, perhaps even breaking, the third harmonic amplitude $(k h=9.8$ behind the shoal) would be significant. For these types of scenarios, an optimized three-layer equation model with a wave breaking scheme could be an option, and the authors are currently developing this model.

The research reported here is partly supported by grants from National Science Foundation (CMS-9528013, CTS-9808542 and CMS 9908392).

Proc. R. Soc. Lond. A (2004) 


\section{Appendix A. Linear-dispersion properties}

The linearized one-dimensional equations in a constant depth can be written in the following dimensional form:

$$
\begin{aligned}
\frac{\partial \zeta}{\partial t}+\delta_{1} h \frac{\partial \boldsymbol{u}_{1}}{\partial x}+\delta_{2} h \frac{\partial \boldsymbol{u}_{2}}{\partial x}+\delta_{3} h^{3} \frac{\partial^{3} \boldsymbol{u}_{1}}{\partial x^{3}}+\delta_{4} h^{3} \frac{\partial^{3} \boldsymbol{u}_{2}}{\partial x^{3}} & =0, \\
\frac{\partial \boldsymbol{u}_{1}}{\partial t}+g \frac{\partial \zeta}{\partial x}+\delta_{5} h^{2} \frac{\partial^{3} \boldsymbol{u}_{1}}{\partial x^{2} t}+\delta_{6} h^{2} \frac{\partial^{3} \boldsymbol{u}_{2}}{\partial x^{2} t} & =0 \\
\boldsymbol{u}_{1}-\boldsymbol{u}_{2}-\delta_{7} h^{2} \frac{\partial^{2} \boldsymbol{u}_{1}}{\partial x^{2}}-\delta_{8} h^{2} \frac{\partial^{2} \boldsymbol{u}_{2}}{\partial x^{2}} & =0,
\end{aligned}
$$

where

$$
\left.\begin{array}{c}
\delta_{1}=-\alpha_{2}, \quad \delta_{2}=1+\alpha_{2}, \quad \delta_{3}=\frac{1}{6}\left(-2 \alpha_{2}^{3}+6 \alpha_{1} \alpha_{2}^{2}-3 \alpha_{1}^{2} \alpha_{2}\right), \\
\delta_{4}=\frac{1}{6}\left(2 \alpha_{2}^{3}-6 \alpha_{1} \alpha_{2}^{2}-6 \alpha_{1} \alpha_{2}+3 \alpha_{3}^{2} \alpha_{2}+6 \alpha_{3} \alpha_{2}+3 \alpha_{3}^{2}+6 \alpha_{3}+2\right), \\
\delta_{5}=\frac{1}{2} \alpha_{1}^{2}-\alpha_{1} \alpha_{2}, \quad \delta_{6}=\alpha_{1} \alpha_{2}+\alpha_{1}, \quad \delta_{7}=-\frac{1}{2}\left(\alpha_{1}^{2}+\alpha_{2}^{2}\right)+\alpha_{1} \alpha_{2}, \\
\delta_{8}=\frac{1}{2}\left(\alpha_{2}^{2}+\alpha_{3}^{2}\right)-\alpha_{1} \alpha_{2}+\alpha_{3}-\alpha_{1} .
\end{array}\right\}
$$

(a) Linear dispersion relation

Substituting the solution form (3.6) into (A 1)-(A 3) yields the linear dispersion relation:

$$
w^{2}=\frac{k^{2} g h\left[1+(k h)^{2} N_{1}+(k h)^{4} N_{2}\right]}{1+(k h)^{2} D_{1}+(k h)^{4} D_{2}}
$$

where

$$
\left.\begin{array}{ll}
N_{1}=\delta_{2} \delta_{7}-\delta_{1} \delta_{8}-\delta_{3}-\delta_{4}, & N_{2}=\delta_{3} \delta_{8}-\delta_{4} \delta_{7} \\
D_{1}=-\delta_{8}-\delta_{5}-\delta_{6}, & D_{2}=\delta_{5} \delta_{8}-\delta_{6} \delta_{7} .
\end{array}\right\}
$$

\section{(b) Derivation of linear shoaling gradient}

The method presented in Schäffer \& Madsen (1995) was applied to the two-layer model. The calculations were performed on a computer using MATHEMATiCA, and the resulting expressions are too tedious to be given here. However, the general solution method is described now. Introducing the solutions

$$
\zeta=a(x) \mathrm{e}^{\mathrm{i} \theta}, \quad \boldsymbol{u}_{1}=\left[\boldsymbol{u}_{1}^{(0)}(x)+\mathrm{i} \hat{\boldsymbol{u}}_{1 x}^{(0)}(x)\right] \mathrm{e}^{\mathrm{i} \theta}, \quad \boldsymbol{u}_{2}=\left[\boldsymbol{u}_{2}^{(0)}(x)+\mathrm{i} \hat{\boldsymbol{u}}_{2 x}^{(0)}(x)\right] \mathrm{e}^{\mathrm{i} \theta},
$$

where

$$
\theta=\omega t-\int k(x) \mathrm{d} x
$$

and $(x)$ indicates variables that are slowly varying quantities of $x$. Note the distinction between $\hat{u}=O\left(h_{x}\right)$ and $u$, the derivative of $\boldsymbol{u}_{n}^{(0)}$. The above solutions are substituted into the linear governing equations, and are truncated to include only up to first derivatives of the slowly varying quantities, i.e. terms such as $h_{x}^{2}, h_{x} k_{x}$, etc., are truncated. To first order (no derivatives of the slowly varying quantities) the system of equations yields the dispersion relation given in Appendix $\mathrm{A} a$.

Proc. R. Soc. Lond. A (2004) 
Working with the second-order components, through numerous substitutions, one can construct a single equation in the form:

$$
S_{1} \frac{a_{x}}{a}+S_{2} \frac{k_{x}}{k}+S_{3} \frac{h_{x}}{h}=0 .
$$

Differentiation of the dispersion relation gives

$$
\frac{k_{x}}{k}=-S_{4} \frac{h_{x}}{h},
$$

which allows us to write the shoaling equation

$$
\frac{a_{x}}{a}=A_{x} \frac{h_{x}}{h},
$$

where

$$
A_{x}=\frac{S_{2} S_{4}-S_{3}}{S_{1}} .
$$

Again, note that the variables $S_{1}, S_{2}, S_{3}$ and $S_{4}$ are determined using symbolicmathematical software and will not be given here.

\section{(c) Second-order nonlinear interactions}

Now we find the nonlinear corrections to the linear problem. The two-layer equations must now be truncated to include quadratic nonlinear terms, as well as linear terms. Collecting the $O\left(\epsilon^{2}\right)$ terms from the substitution of the assumed steady wave, (3.6), into the nonlinear-equation system will yield an equation system in the general form

$$
\left[\begin{array}{ccc}
b_{11} & b_{12} & b_{13} \\
b_{21} & b_{22} & b_{23} \\
0 & b_{32} & b_{33}
\end{array}\right]\left(\begin{array}{c}
\zeta^{(1)} \\
\boldsymbol{u}_{1}^{(1)} \\
\boldsymbol{u}_{2}^{(1)}
\end{array}\right)=\left[\begin{array}{l}
R_{1} \\
R_{2} \\
R_{3}
\end{array}\right],
$$

where $b_{11}, \ldots, b_{33}$ are functions of the linear $\delta$ coefficients, and $R_{1}, \ldots, R_{3}$ are tedious functions of the $\alpha$ and $\beta$ parameters.

To find the sub- and super-harmonic amplitudes for the bichromatic wave group problem, the procedure is the same as described above for the steady wave (single, first-order harmonic) problem. The assumed solution (3.18) is substituted into the two-layer equation system. For each of the forced second-order solutions, $\left[\left(k_{1}-k_{2}\right) x-\left(w_{1}-w_{2}\right) t\right]$ and $\left[\left(k_{1}+k_{2}\right) x+\left(w_{1}-w_{2}\right) t\right]$, the matrix system is written in the same form as for the steady wave problem. The expressions for the nonlinear harmonics are too tedious to include in the published manuscript, but can be obtained through the first author via email at plynett@civil.tamu.edu.

\section{Appendix B. Heuristic analysis of truncation error}

In this section, the truncation error of the two-layer model equations is examined, and compared with those of existing one-layer depth-integrated model equations. Additionally, minimization of the two-layer truncation error yields an estimate of the layer interface location, and this value can be compared with the interface location determined in $\S 3 f$. It is noted that the form of the truncation error in the present 


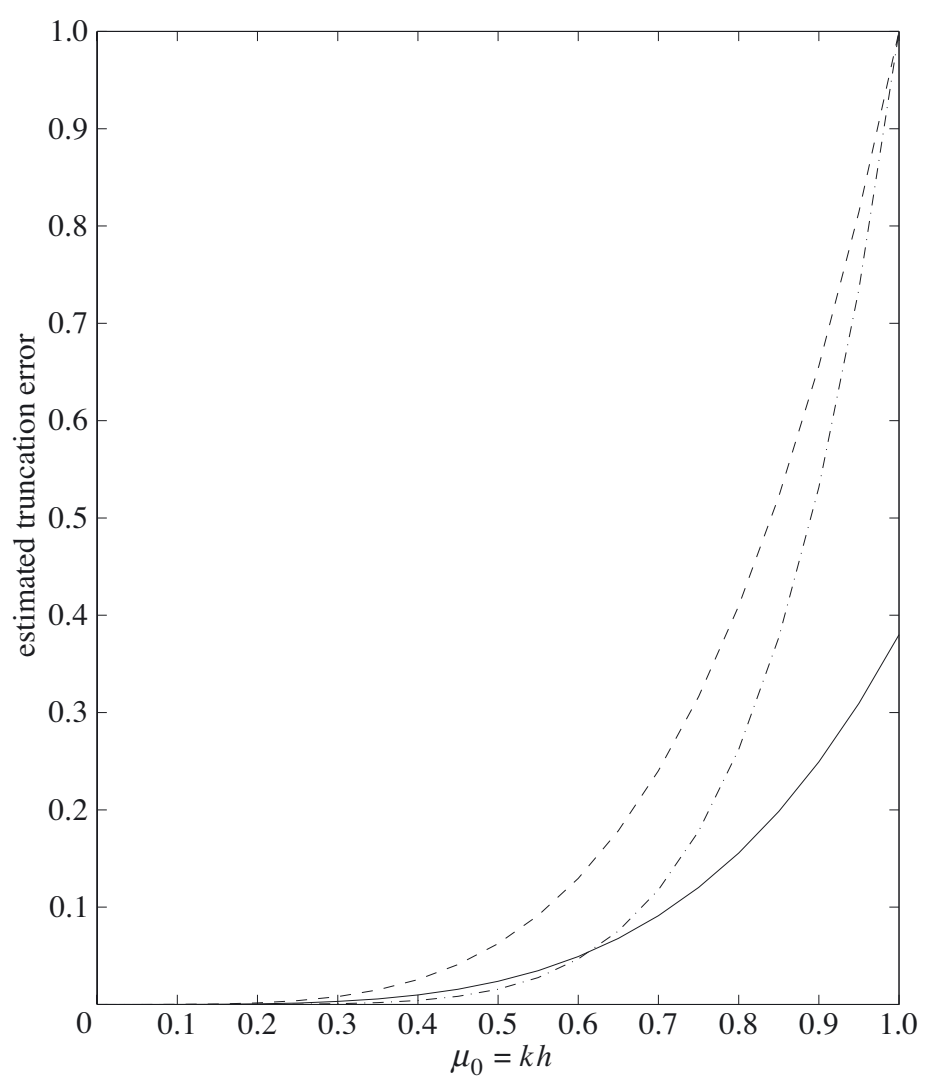

Figure 14. Comparison of the truncation errors for three different models: $\mu_{0}^{4}$ Boussinesq equations (dash-dashed line), $\mu_{0}^{6}$ high-order Boussinesq equations (dot-dashed line), $0.38 \mu_{0}^{4}$ minimized error for two-layer equations (solid line).

model equations is a direct result of the scaling used to non-dimensionalize the Euler equations. From (2.32)-(2.34), the overall accuracy of the present model equations, or the largest truncation errors of the three equations, can be expressed as $O\left(\mu_{0}^{2} \mu_{1}^{2}, \mu_{2}^{4}\right)$. The heuristic approach taken here is to look at this truncation error as a finite value, not an order, and use this value to estimate the accuracy of the model compared with the $O\left(\mu_{0}^{2}\right)$ Boussinesq equations (Nwogu 1993) and the high-order, $O\left(\mu_{0}^{4}\right)$ Boussinesqtype equations (Gobbi et al. 2000).

The truncation error of the present two-layer model equations has an upper bound of $O\left(\mu_{0}^{4}\right)$ when either $d_{1}$ or $d_{2}$ is equal to $h_{0}$, which corresponds to the truncation error of a traditional (one-layer) Boussinesq model. The lower bound occurs when $\mu_{0}^{2} \mu_{1}^{2}=\mu_{2}^{4}$, or $d_{1} h_{0}=d_{2}^{2}$, which gives $d_{1}=0.38 h_{0}, d_{2}=0.62 h_{0}$. We expect that the two-layer model will yield a more accurate result than the one-layer model, due to the fact that the error of approximation for the two-layer model is smaller. The truncation error values for these two models, as well as the high-order Boussinesqtype equations that have a truncation error of $O\left(\mu_{0}^{6}\right)$, are shown in figure 14 . This plot indicates that, over the range $\mu_{0}<0.6$, the high-order Boussinesq model should yield results slightly more accurate than those of the two-layer model. Over the range $\mu_{0}>0.6$, however, the two-layer model should be significantly more accurate 
than the high-order model. In fact, compared with a single-layer model of any order, $O\left(\mu_{0}^{n}\right)$, the two-layer model should achieve higher accuracy as $\mu_{0}$ approaches 1 .

In the present two-layer model, the truncation error tells us that $\mu_{0}^{2} \mu_{1}^{2}$ and $\mu_{2}^{4}$ should be small compared with the included terms, which, due to the appearance of both $O\left(\mu_{0}^{2}\right)$ and $O\left(\mu_{1}^{2}\right)$ in the momentum equations, still requires that both $O\left(\mu_{0}^{2}\right)$ and $O\left(\mu_{1}^{2}\right) \ll 1$. The restriction of $O\left(\mu_{0}^{2}\right) \ll 1$ could be avoided by including $O\left(\mu_{0}^{2} \mu_{1}^{2}\right)$ terms, thereby making the truncation error of the model $O\left(\mu_{0}^{2} \mu_{1}^{4}, \mu_{1}^{4}, \mu_{2}^{4}\right)$. However, inclusion of $O\left(\mu_{0}^{2} \mu_{1}^{2}\right)$ terms yields a model with fifth order in space derivatives. This is unacceptable for this particular derivation, whose primary goal is to create a highorder accurate model without requiring high-order derivatives.

\section{References}

Agnon, Y., Madsen, P. A. \& Schäffer, H. 1999 A new approach to high order Boussinesq models. J. Fluid Mech. 399, 319-333.

Berkoff, J. C. W., Booij, N. \& Radder, A. C. 1982 Verification of numerical wave propagation models for simple harmonic linear water waves. Coastal Engng 6, 255-279.

Chen, Q., Kirby, J. T., Dalrymple, R. A., Kennedy, A. B. \& Chawla, A. 2000 Boussinesq modeling of wave transformation, breaking, and runup. Part I. 2D. J. Wtrwy Port Coastal Ocean Engng 126, $57-62$.

Dingemans, M. 1994 Comparison of computations with Boussinesq-like models and laboratory measurements. Mast-G8M note, H1684. Delft Hydraulics.

Gobbi, M. F. \& Kirby, J. T. 1999 Wave evolution over submerged sills: tests of a high-order Boussinesq model. Coastal Engng 37, 57-96.

Gobbi, M. F., Kirby, J. T. \& Wei, G. 2000 A fully nonlinear Boussinesq model for surface waves. II. Extension to $O\left((k h)^{4}\right)$. J. Fluid Mech. 405, 182-210.

Hsiao, S.-C. \& Liu, P. L.-F. 2002 Nonlinear water waves propagating over a permeable bed. Proc. R. Soc. Lond. A 485, 1291-1322.

Israeli, M. \& Orszag, S. A. 1981 Approximation of radiation boundary conditions. J. Comput. Phys. 41, 113-115.

Kanayama, S., Tanaka, H. \& Shuto, N. 1998 A multi-level model for nonlinear dispersive water waves. In Coastal engineering (ed. B. L. Edge), vol. 1, pp. 576-588. Reston, VA: American Society of Civil Engineers.

Kennedy, A. B., Kirby, J. T., Chen, Q. \& Dalrymple, R. A. 2001 Boussinesq-type equations with improved nonlinear behaviour. Wave Motion 33, 225-243.

Kirby, J. T., Wei, G., Chen, Q., Kennedy, A. B. \& Dalrymple, R. A. 1998 Funwave 1.0: fully nonlinear Boussinesq wave model documentation and user's manual. Center for Applied Coastal Research, University of Delaware, Newark, DE, USA.

Liu, P. L.-F. 1994 Model equations for wave propagation from deep to shallow water. In Advances in coastal engineering (ed. P. L.-F. Liu), vol. 1, pp. 125-157. World Scientific.

Liu, P. L.-F., Yoon, S. B. \& Kirby, J. T. 1985 Nonlinear refraction-diffraction of waves in shallow water. J. Fluid Mech. 153, 184-201.

Lynett, P. 2002 A multi-layer approach to modeling nonlinear, dispersive waves from deep water to the shore. PhD thesis, Cornell University, Ithaca, NY, USA.

Lynett, P. \& Liu, P. L.-F. 2002 A numerical study of submarine-landslide-generated waves and run-up. Proc. R. Soc. Lond. A 458, 2885-2910.

Madsen, P. A. \& Schäffer, H. A. 1998 Higher order Boussinesq-type equations for surface gravity waves: derivation and analysis. Phil. Trans. R. Soc. Lond. A 356, 3123-3181.

Madsen, P. A. \& Sørensen, O. R. 1992 A new form of the Boussinesq equations with improved linear dispersion characteristics. II. A slowly varying bathymetry. Coastal Engng 18, 183-204.

Proc. R. Soc. Lond. A (2004) 
Madsen, P. A., Bingham, H. B. \& Liu, H. 2002 A new Boussinesq method for fully nonlinear waves from shallow to deep water. J. Fluid Mech. 462, 1-30.

Madsen, P. A., Bingham, H. B. \& Schäffer, H. A. 2003 Boussinesq-type formulations for fully nonlinear and extremely dispersive water waves: derivation and analysis. Proc. R. Soc. Lond. A 459, 1075-1104.

Nwogu, O. 1993 Alternative form of Boussinesq equations for nearshore wave propagation. $J$. Wtrwy Port Coastal Ocean Engng 119, 618-638.

Peregrine, D. H. 1967 Long waves on a beach. J. Fluid Mech. 27, 815-827.

Schäffer, H. A. 1996 Second-order wavemaker theory for irregular wave. Ocean Engng 23, 47-88.

Schäffer, H. A. \& Madsen, P. A. 1995 Further enchancements of Boussinesq-type equations. Coastal Engng 26, 1-14.

Tanaka, M. 1986 The stability of solitary waves. Phys. Fluids 29, 650-655.

Vincent, C. L. \& Briggs, M. J. 1989 Refraction-diffraction of irregular waves over a mound. J. Wtrwy Port Coastal Ocean Engng 115, 269-284.

Wei, G. \& Kirby, J. T. 1995 A time-dependent numerical code for extended Boussinesq equations. J. Wtrwy Port Coastal Ocean Engng 120, 251-261.

Wei, G., Kirby, J. T., Grilli, S. T. \& Subramanya, R. 1995 A fully nonlinear Boussinesq model for surface waves. I. Highly nonlinear unsteady waves. J. Fluid Mech. 294, 71-92.

Woo, S-B. \& Liu, P. L.-F. 2001 A Petrov-Galerkin finite element model for one-dimensional fully non-linear and weakly dispersive wave propagation. Int. J. Numer. Meth. Engng 37, $541-575$.

As this paper exceeds the maximum length normally permitted, the authors have agreed to contribute to production costs. 
